\title{
Working towards recalcitrance mechanisms: increased xylan and homogalacturonan production by overexpression of GAlactUronosy/Transferase 12 (GAUT12) causes increased recalcitrance and decreased growth in Populus
}

\author{
Ajaya K. Biswal 1,3,4, Melani A. Atmodjo 1,3,4, Sivakumar Pattathil ${ }^{3,4,8}$, Robert A. Amos ${ }^{1,3,4}$,
}

Xiaohan Yang 4,5, Kim Winkeler 4,6, Cassandra Collins ${ }^{4,6}$, Sushree S. Mohanty 3,4, David Ryno 3,4, Li Tan 1,3,4, Ivana Gelineo-Albersheim ${ }^{3,4}$, Kimberly Hunt ${ }^{3,4,9}$, Robert W. Sykes ${ }^{4,7,10}$, Geoffrey B. Turner ${ }^{4,7,11}$, Angela Ziebell ${ }^{4,7}$, Mark F. Davis $^{4,7}$, Stephen R. Decker ${ }^{4,7}$, Michael G. Hahn ${ }^{1,2,4}$ and Debra Mohnen ${ }^{1,3,4^{*}}$

\begin{abstract}
Background: The development of fast-growing hardwood trees as a source of lignocellulosic biomass for biofuel and biomaterial production requires a thorough understanding of the plant cell wall structure and function that underlie the inherent recalcitrance properties of woody biomass. Downregulation of GAUT12.1 in Populus deltoides was recently reported to result in improved biomass saccharification, plant growth, and biomass yield. To further understand GAUT12.1 function in biomass recalcitrance and plant growth, here we report the effects of P. trichocarpa GAUT12.1 overexpression in P. deltoides.

Results: Increasing GAUT12.1 transcript expression by 7-49\% in P. deltoides PtGAUT12.1-overexpression (OE) lines resulted in a nearly complete opposite biomass saccharification and plant growth phenotype to that observed previously in PdGAUT12.1-knockdown (KD) lines. This included significantly reduced glucose, xylose, and total sugar release (12-13\%), plant height (6-54\%), stem diameter (8-40\%), and overall total aerial biomass yield (48-61\%) in 3-monthold, greenhouse-grown PtGAUT12.1-OE lines compared to controls. Total lignin content was unaffected by the gene overexpression. Importantly, selected PtGAUT12.1-OE lines retained the recalcitrance and growth phenotypes upon growth for 9 months in the greenhouse and 2.8 years in the field. PtGAUT12.1-OE plants had significantly smaller leaves with lower relative water content, and significantly reduced stem wood xylem cell numbers and size. At the cell wall level, xylose and galacturonic acid contents increased markedly in total cell walls as well as in soluble and insoluble cell wall extracts, consistent with increased amounts of xylan and homogalacturonan in the PtGAUT12.1-OE lines. This led to increased cell wall recalcitrance, as manifested by the 9-15\% reduced amounts of recovered extractable wall materials and 8-15\% greater amounts of final insoluble pellet in the PtGAUT12.1-OE lines compared to controls.
\end{abstract}

\footnotetext{
*Correspondence: dmohnen@ccrc.uga.edu

${ }^{3}$ Complex Carbohydrate Research Center, University of Georgia, 315

Riverbend Rd., Athens, GA 30602-4712, USA

Full list of author information is available at the end of the article
} 
Conclusions: The combined phenotype and chemotype data from P. deltoides PtGAUT12.1-OE and PdGAUT12.1-KD transgenics clearly establish GAUT12.1 as a recalcitrance- and growth-associated gene in poplar. Overall, the data support the hypothesis that GAUT12.1 synthesizes either an HG-containing primer for xylan synthesis or an HG glycan required for proper xylan deposition, anchoring, and/or architecture in the wall, and the possibility of HG and xylan glycans being connected to each other by a base-sensitive covalent linkage.

Keywords: Plant cell wall, Biofuel, Biomass, Pectin, Xylan, Yield

\section{Background}

Development of strategies to deconstruct lignocellulosic biomass for biofuels and biomaterials production is essential to advance a sustainable economy and to mitigate greenhouse gas emission-related climate change [1, 2]. Trees accumulate a major portion of terrestrial biomass as secondary cell walls that account for a substantial amount of global carbon sequestration [3, 4]. Hardwood biomass is a complex polymeric matrix of cellulose, hemicellulose (primarily xylan), and lignin along with significant amounts of pectin $[5,6]$. The structural diversity of the different wall polymers, and the inter- and intramolecular interactions via covalent and non-covalent linkages among them, influence the mechanical and chemical properties of the biomass that are important for tree survival and for quality (e.g., fiber length, fiber strength) of wood-derived materials such as timber, paper, cellulose, lignin, and others $[7,8]$. Understanding the structural complexity, interaction, and functionality of the cell wall polymers is therefore essential for unraveling the molecular basis of biomass recalcitrance and plant growth, and to generate by biotechnological manipulation improved biomass with reduced recalcitrance and high yield.

In prior research, we identified a recalcitrance-associated gene, GAlactUronosylTransferase (GAUT)12 whose modified expression in poplar led to both reduced biomass recalcitrance and increased plant growth [5]. GAUT12 is a putative galacturonosyltransferase (GalAT) belonging to the GAUT gene family (Fig. 1) within the glycosyltransferase (GT) 8 family [9, 10]. GAUT12 was first identified as a gene involved in Arabidopsis thaliana $(A t)$ secondary wall formation $[11,12]$. It is highly expressed in stems and roots, particularly in cells undergoing secondary wall thickening including interfascicular fibers and primary and secondary xylem $[11,13]$. Arabidopsis irregular xylem8 (irx8) mutants, defective in the GAUT12 gene $[11,12]$ are severely dwarfed, semi-sterile due to indehiscent anthers and have a collapsed xylem vessel phenotype [13-15]. Compared to wild type (WT), Arabidopsis irx8 mutant cell walls have a greater than $50 \%$ reduction in glucuronoxylan $(\mathrm{GX})$ and an almost complete absence of the $\beta$-D-Xylp-(1,3)- $\alpha$-L-Rhap-(1,2)$\alpha-\mathrm{D}-\mathrm{Gal} p \mathrm{~A}-(1,4)-\mathrm{D}-\mathrm{Xyl} p$ xylan reducing end tetrasaccharide sequence, indicating a critical role of AtGAUT12 in

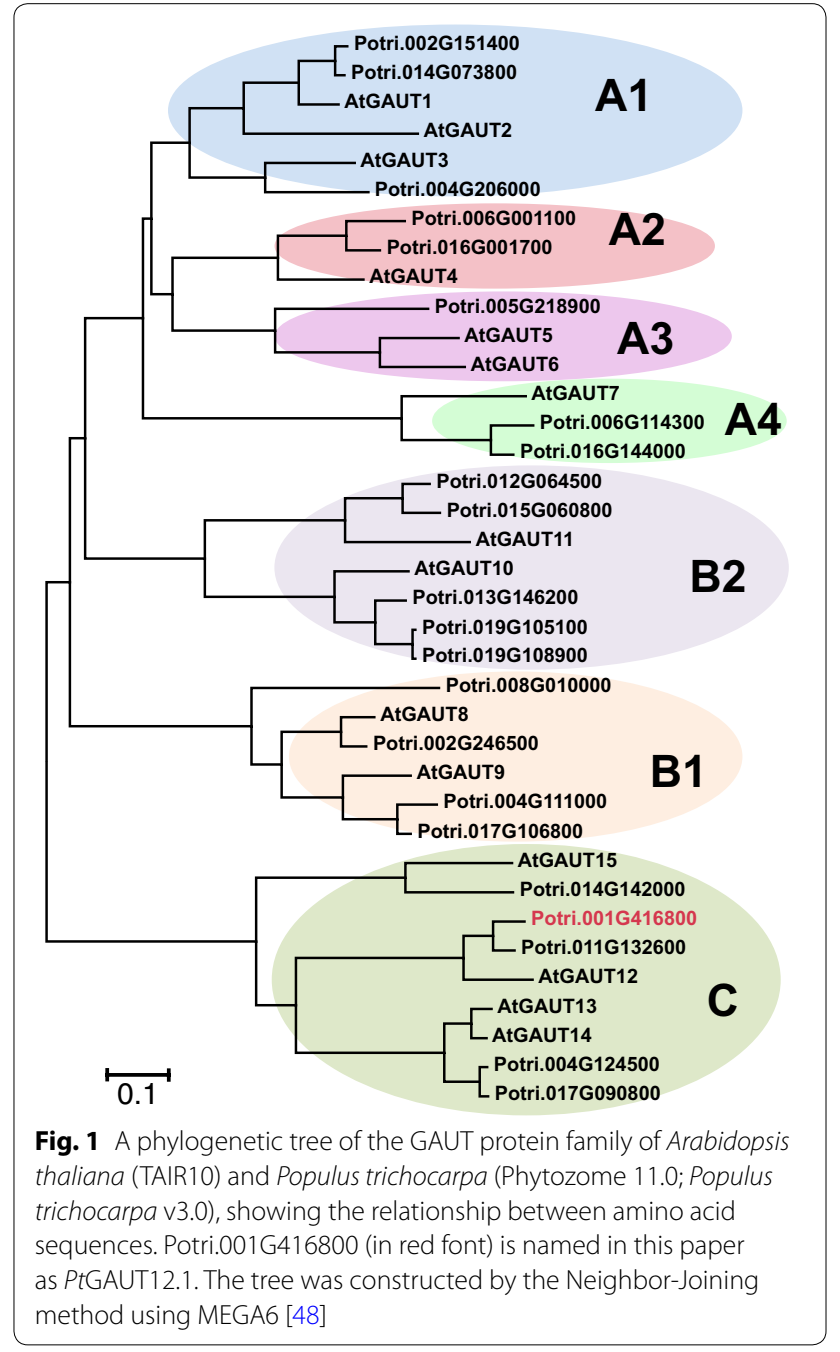

xylan biosynthesis $[11,13,14,16]$. However, significantly decreased amounts of pectin were also observed in pectin-enriched wall fractions from ir $x 8$ mutants compared to WT $[14,16]$, leading to the hypothesis that AtGAUT12 is involved in either the insertion of GalA into the xylan reducing end sequence, or in the synthesis of a subfraction of homogalacturonan (HG) [14] required for xylan synthesis. Arabidopsis stem lignin content was also reduced in the irx 8 mutants, and immunohistochemistry of stem sections using multiple anti-xylan monoclonal 
antibodies revealed different xylan localization patterns between the irx 8 mutants and WT $[15,17]$, suggesting a role for the GAUT12-synthesized cell wall polymer in wall architecture. Based on the data from Arabidopsis, the results suggest that GAUT12 functions in the synthesis of a structure required for xylan and lignin deposition during secondary cell wall formation in Arabidopsis, and that the structure either contains, or is dependent upon, an HG-containing glycan.

Since several other members of the GAUT gene family have been shown to have homogalacturonan:galactur onosyltransferase (HG:GalAT) activity [10, 18], GAUT12 was hypothesized to also have GalAT activity. GAUT12 is predicted to be a type II membrane protein and has been shown to localize to the Golgi in both Arabidopsis and poplar $[13,19]$. In a study designed to identify the enzyme function of GAUT12, it was shown that the Arabidopsis ir $x 8$ mutant did not have reduced xylan xylosyltransferase (XylT) or xylan glucuronosyltransferase (GlcAT) activities [16, 20], thereby providing no support for a function of GAUT12 directly in xylan synthesis. On the contrary, Hao et al. [15] identified $\sim 45 \%$ reduced HG:GalAT activity in microsomes from irx8/gaut 12 stems compared to WT, suggesting a possible function of GAUT12 in HG synthesis. However, no HG:GalAT activity was detected from GAUT12-immunoabsorbed from WT solubilized microsomes [15] when a typical HG:GalAT enzyme assay was used [10, 21]. While it is possible that the standard HG:GalAT reaction conditions (e.g., exogenous acceptor used) and/or the amount or condition of the immunopurified Arabidopsis GAUT12 was insufficient to detect HG:GalAT activity in vitro from the immunopurified Arabidopsis GAUT12, the role of GAUT12 in xylan biosynthesis remains to be determined.

Poplar has two homologs of AtGAUT12, designated GAUT12.1 (Potri.001G416800, Genemodel V3.0; Phytozome 12.0) and GAUT12.2 (Potri.011G132600, Genemodel V3.0; Phytozome 12.0) that are 91 and $90 \%$ identical to each other in their amino acid and nucleotide sequences, respectively. Both genes are expressed in poplar stem primary and differentiating xylem, secondary xylem, and phloem fibers, with GAUT12.1 expression being seven times greater than GAUT12.2 [19, 22]. Simultaneous downregulation of both genes in Populus trichocarpa [22] and Populus alba $x$ tremula [23] significantly reduced the transcript level of both genes and resulted in $20-40 \%$ decreased stem xylan content compared to controls, consistent with a function of GAUT12 in xylan biosynthesis. The xylan reducing end tetrasaccharide sequence was also reduced in the GAUT12 knockdown (KD) transgenics compared to WT in the P. alba $x$ tremula study [23]. However, in contrast to Arabidopsis-dwarfed ir $x 8$ mutants, the transgenic double
GAUT12.1/GAUT12.2-knocked down poplar plants did not show reduced growth or collapsed xylem phenotypes, although they had thinner cell walls and, in one study, slightly deformed vessel cells [22, 23]. Furthermore, lignin content was increased in the $P$. trichocarpa GAUT12-KD wood biomass [22], but was reduced in the P. alba $x$ tremula GAUT12-KD samples [23].

Recently, we reported the specific downregulation of only the GAUT12.1 gene in P. deltoides [5] and described the consequences of this genetic manipulation on plant/ wood growth and development and biomass saccharification efficiency. PdGAUT12.1 was selected due to its greater transcript abundance than PdGAUT12.2. As expected, the cell walls of PdGAUT12.1-KD plants were significantly reduced in xylose (Xyl) and galacturonic acid (GalA) content, in comparison to control plants. These results indicated that PdGAUT12.1 is involved in xylan and pectin formation in Populus, in a similar fashion to AtGAUT12 in Arabidopsis. Wood from the PdGAUT12.1-KD lines also had reduced recalcitrance compared to control lines [5]. In agreement with this finding, a recent study of segregating Eucalyptus hybrid tree populations using network-based data integration methodology revealed the association of GAUT12 with sugar release traits [24]. Contrary to the $P$. trichocarpa and $P$. alba $x$ tremula double homolog knockdown transgenics described above, however, PdGAUT12.1-KD lines showed no change in the total lignin content [5]. Most importantly, PdGAUT12.1-KD plants had larger cell size, growth, and biomass yield compared to the WT [5], which is in contrast to the negative or neutral growth phenotypes of the Arabidopsis irx 8 knockout mutants and the poplar double homolog knockdown transgenics $[22,23]$. Overall, the results support the hypothesis that GAUT12 is required for the synthesis of a native xylancontaining polymer, but also suggest that there is a fine balance between the amount and/or structure of that polymer, wall structural properties and plant growth.

Despite the above-described extensive research on the gaut12/irx 8 mutants and the GAUT12 gene and transgenics to date, the exact biochemical and biological function of GAUT12 remains unsolved. It is also unclear why the lack of GAUT12 expression inhibits growth so severely in Arabidopsis irx8 knockout mutants [11-14], but the simultaneous reduced expression of GAUT12.1 and GAUT12.2 in poplar does not negatively impact growth [22, 23] and the reduced expression of only GAUT12.1 increases growth in P. deltoides [5].

The goal of the research reported here was to understand the biological function of GAUT12 in poplar wood, and the mechanism for how modified GAUT12 expression affects biomass recalcitrance and growth. To this end, we overexpressed P. trichocarpa GAUT12.1 
(PtGAUT12.1) in P. deltoides and characterized the transgenic plants for recalcitrance and growth phenotypes. We hypothesized that in the resulting PtGAUT12.1 overexpression (OE) lines, we would obtain one of the two results: (1) GAUT12 enzyme function would require coordinated expression of multiple genes, in which case overexpression of GAUT12 alone would not increase the expression of the synthesized polymers and therefore no recalcitrance/growth phenotype would be manifested, or (2) GAUT12 overexpression would increase the amount of GAUT12-synthesized polymers, resulting in associated phenotypes/chemotypes of the plant and cell walls. The latter possibility would enable the analyses of such modified cell walls with the goal of obtaining further insight into the biological and cell wall/enzyme function of GAUT12. Here we report that overexpression of PtGAUT12.1 yields PtGAUT12.1-OE lines with the opposite growth, recalcitrance, and cell wall phenotypes as those observed in the P. deltoides GAUT12.1knockdown (PdGAUT12.1-KD) lines [5]. Analysis of the PtGAUT12.1-OE biomass and phenotypes, and comparison of these results with the chemotypes/phenotypes of the previously reported PdGAUT12.1-KD data provide a comprehensive dataset that strongly supports the hypothesis that GAUT12 functions in the synthesis of a xylan- and homogalacturonan-containing polymer that has roles in cell wall integrity, biomass recalcitrance, and plant growth in woody feedstock.

\section{Results}

Populus GAUT12.1 is expressed in the shoot apex, young developing leaves, and internodes, as well as in secondary wall-rich stem and root tissues, indicating a broader role for GAUT12 than only in secondary walls

Previous studies of GAUT12 primarily emphasized its function in secondary cell walls, focusing mostly on stem tissues (in Arabidopsis) [13-16] and woody biomass (in poplar) $[5,22,23]$. High GAUT12 expression was found in xylem while low levels of expression were found in tissues such as anther, pollen, leaf vascular tissue, and hypocotyls $[11,14,15]$. Low expression of Pt and PdGAUT12.1 was also reported in poplar leaves [5, 22]. To confirm the broad expression of GAUT12 and to dissect its expression in young tissues, we analyzed PdGAUT12.1 and PdGAUT12.2 expression in developing organs of $P$. deltoides by quantitative RT-PCR. Xylem tissues were included as a control. As expected, xylem tissues had the highest levels of expression of both the PdGAUT12.1 and PdGAUT12.2 genes (Fig. 2C, D). However, PdGAUT12.1 expression was also evident, albeit at much lower levels, in the phloem and root tissues, and expression was detected at lower but clearly measurable levels in the apex, the first three leaves, and the first three internodes from the top of the plant (Fig. 2C). Similar but lower expression was observed for PdGAUT12.2, although no expression was detectable in leaves 1, 2, and 3 (Fig. 2D).

We also studied PdGAUT12.1 expression in the different developmental zones of poplar wood by RNA blot analysis using a $3^{\prime}$-UTR nucleotide gene probe (Fig. 2E, F). PdGAUT12.1 was expressed very strongly in the secondary wall formation zone, and also in a lower but substantial amount in the vascular cambium. Much lower expression was detected in the expansion, transition, and cell death zones as well as in whole stem tissue. No PdGAUT12.1 expression was detectable in phloem and leaf tissues by this RNA blot method.

\section{Overexpression of PtGAUT12.1 in Populus deltoides}

At the conception of this work, the sequenced $P$. trichocarpa genome was available and used as the poplar reference genome within the BioEnergy Science Center (BESC). The genome information of $P$. deltoides, the poplar species used as genetic background for transgenesis in BESC, was at that time unavailable. Therefore, we decided to clone and overexpress $P$. trichocarpa GAUT12.1 in P. deltoides. Both the P. deltoides GAUT12.1 and GAUT12.2 were later cloned for the purpose of complementation of Arabidopsis irx 8 mutants, the results of which have been reported previously [5]. Comparison of P. trichocarpa GAUT12.1 and GAUT12.2 with their cloned $P$. deltoides counterparts (Fig. 2A, B) showed that both sets of orthologs share $99 \%$ sequence identity at both the protein and nucleotide levels. Recently, a pre-released version of $P$. deltoides genome has become available through Phytozome 12 (https://phytozome.jgi. doe.gov). It is noteworthy, however, that while Phytozome Podel.11G130300.1 coding sequence matches 99\% to the cloned PdGAUT12.2, Podel.01G434500.1 coding sequence seems to be incomplete, and thus matches the cloned PdGAUT12.1 by only $94 \%$. Based on the relatively high sequence similarity between the PtGAUT12.1 and PdGAUT12.1, we expected that overexpression of the former in $P$. deltoides would produce similar phenotypic effects as would overexpression of the latter.

An overexpression construct containing $P$. trichocarpa GAUT12.1 coding sequence (1602 bp) driven by A. thaliana Ubiquitin3 promoter (Fig. 3A-C) was introduced into the $P$. deltoides clone WV94 background. Thirteen PtGAUT12.1-overexpression (OE) transgenic lines (AB29.1 through AB29.13) were generated with the presence of the transgene confirmed by PCR in each of the lines (data not shown). For this study, 10-15 clones of each of the thirteen PtGAUT12.1-OE lines were analyzed along with 25 non-transformed wild-type (WT) plants and 10-15 clones of eight independent vector control lines (V. Control-1 through 8). 


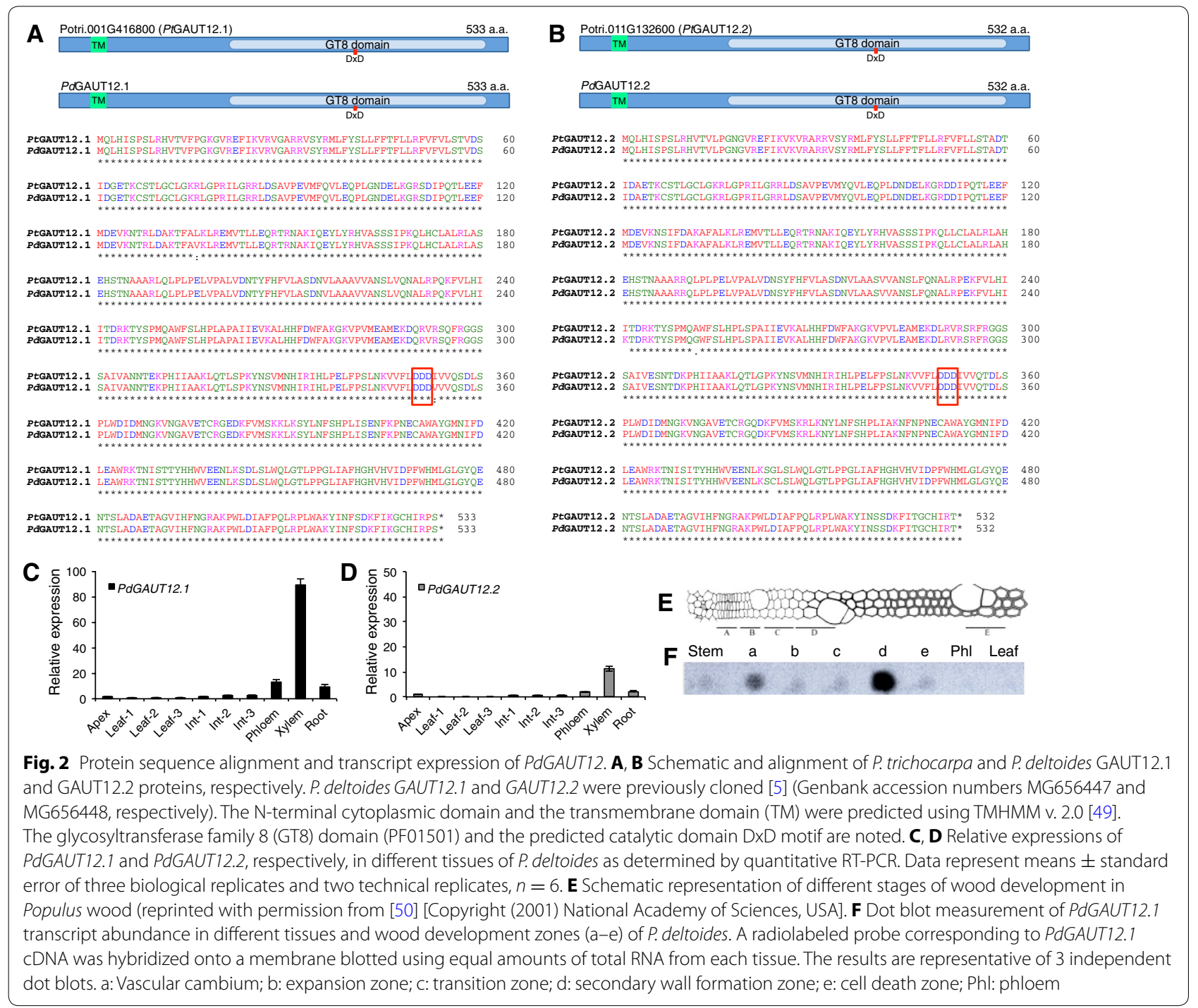

GAUT12.1 and GAUT12.2 transcript expression in the PtGAUT12.1-OE lines compared to controls was investigated using quantitative RT-PCR with primer pairs matching both endogenous PdGAUT12.1 and the transgene PtGAUT12.1. Total GAUT12.1 transcript expression was increased by $7-49 \%$ in all thirteen $\mathrm{OE}$ lines compared to the WT and vector controls (Fig. 3D). Based on the extent of transcript overexpression, the PtGAUT12.1-OE lines were divided into three groups: lines AB29.1, AB29.8, AB29.10, and AB29.11 had 7-9\% increased transcript levels; lines AB29.3, AB29.4, AB29.5, AB29.6, and AB29.13 lines had 12-20\% increased transcript levels; and AB29.2, AB29.7, AB29.9, and AB29.12 lines had 31-49\% increased GAUT12.1 transcript levels compared to the controls. As expected, PdGAUT12.2 transcript expression in the $\mathrm{OE}$ lines was not affected by PtGAUT12.1 overexpression and remained comparable to the controls (Fig. 3D).

\section{PtGAUT12.1 overexpression inhibits saccharification but does not affect total lignin content}

The effect of PtGAUT12.1 overexpression on sugar release from $P$. deltoides wood was determined by subjecting wood biomass samples from 9-month-old control and PtGAUT12.1-OE trees to hot water pretreatment and enzymatic hydrolysis. Eight of the thirteen PtGAUT12.1-OE lines had 4-12\% significantly decreased glucose release per gram dry biomass compared to WT and vector controls (Fig. 4A, Additional file 1A). Significant decreases were also observed for xylose release 


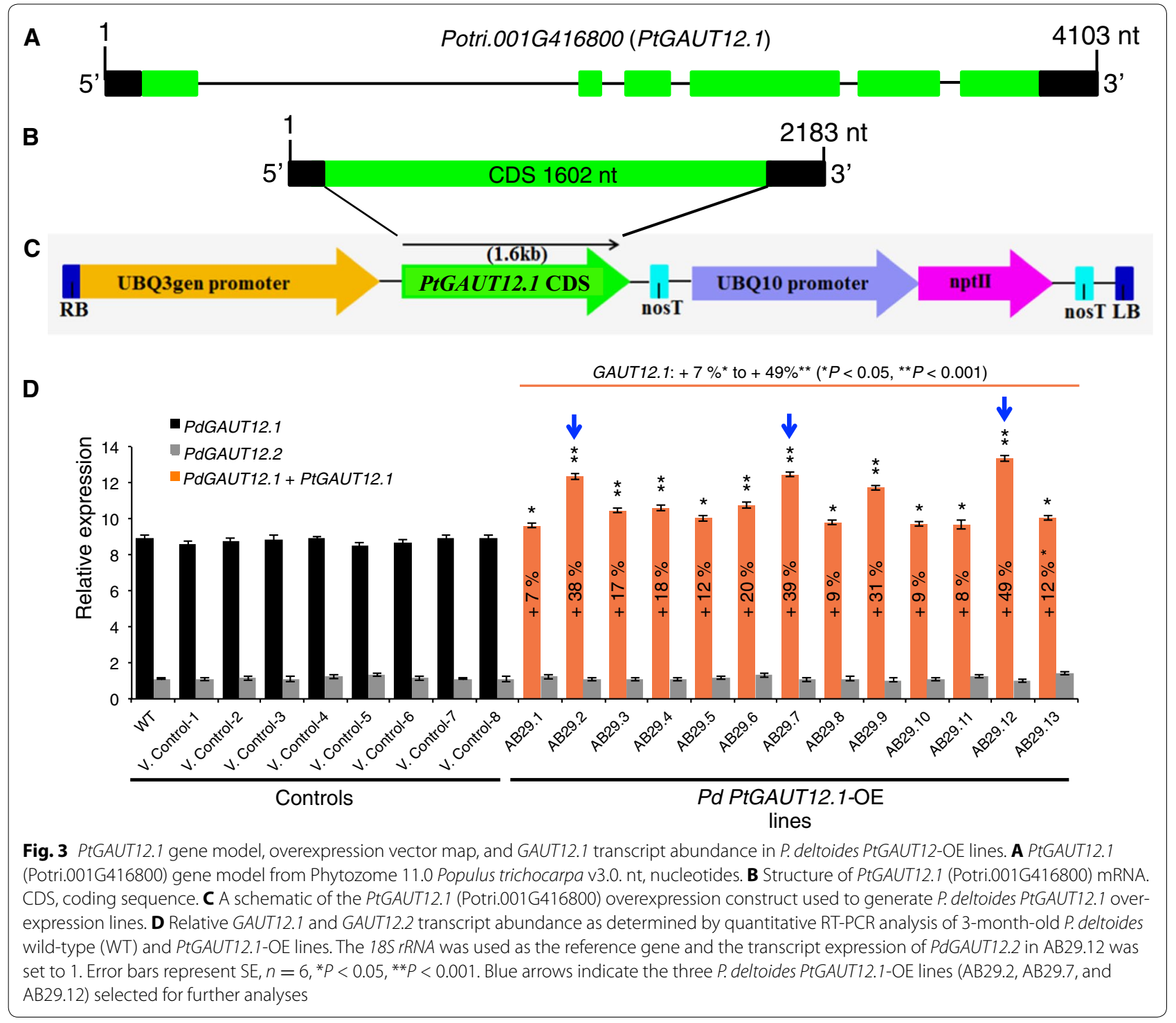

(5-13\%; Fig. 4B, Additional file 1B) and total sugar release (4-12\%; Fig. 4C, Additional file 1C) per gram dry biomass in six and five, respectively, of the thirteen transgenic lines compared to controls.

We analyzed the amount of lignin in wood samples from all control and PtGAUT12.1-OE lines by pyrolysis molecular beam mass spectrometry. Total lignin content in the PtGAUT12.1-OE lines (25.2-26.3\% in AB29.1AB29.13) was similar to that in WT (25.7\%) and vector controls (24.7-26.7\% in V. Control-1 to -8) (Fig. 5A, Additional file 2). However, the lignin syringyl-to-guaiacyl $(\mathrm{S} / \mathrm{G})$ ratios were significantly decreased $(8-11 \%)$ in five of the thirteen PtGAUT12.1-OE lines compared to those of WT and vector controls (Fig. 5B, Additional file 2).

\section{PtGAUT12.1 overexpression decreases plant growth} and biomass yield in $P$. deltoides

Assessment of the effects of PtGAUT12.1 overexpression on plant growth was first carried out on 3-monthold greenhouse-grown plants. Inhibition of vegetative plant growth was observed in the PtGAUT12.1-OE plants compared to the controls (Fig. 6A). Nine of the thirteen PtGAUT12.1-OE lines showed 6-54\% significantly reduced plant height and $8-40 \%$ reduced stem radial diameter compared to $\mathrm{WT}$ and vector controls (Fig. 6B, C, Additional file 3). Both PtGAUT12.1-OE plant height and stem diameter were negatively correlated with the total GAUT12.1 transcript expression (Additional file 4). This growth inhibition led to an 

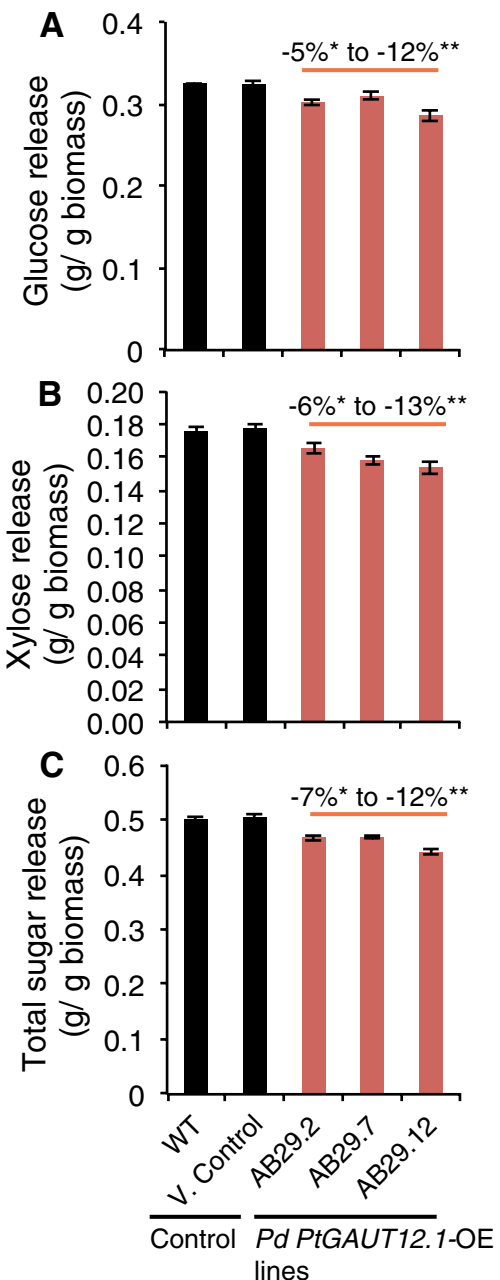

Fig. 4 Saccharification yield of $P$. deltoides PtGAUT12.1-overexpression lines. A Glucose, $\mathbf{B}$ xylose, and $\mathbf{C}$ total sugar release from $P$. deltoides WT, vector control, and PtGAUT12.1-OE lines. Data are mean \pm SE, $n=25$ for WT, $n=120$ for vector control (eight different insertion vector control lines, each with $n=15$, see Additional file 1 for the full dataset), and $n=10-15$ for the PtGAUT12.1-OE lines. Statistical analysis was by one-way analysis of variance (ANOVA) followed by Tukey's multiple comparison test using Statistica 5.0

overall $48-61 \%$ decrease in total aerial dry biomass of the greenhouse-grown, 3-month-old PtGAUT12.1-OE plants (Fig. 6D).

Three PtGAUT12.1-OE lines with the greatest increase in GAUT12.1 transcript expression (AB29.2, AB29.7, and AB29.12; Fig. 3D) were selected for additional growth assessment in the greenhouse. Throughout the 9-month growing period, the three PtGAUT12.1-OE lines continued to exhibit reduced growth characteristics, including a $48-54 \%$ reduction in plant height and a $32-40 \%$ reduction in stem diameter compared to controls (Fig. 6E, F). Except for the field trial, further studies reported here

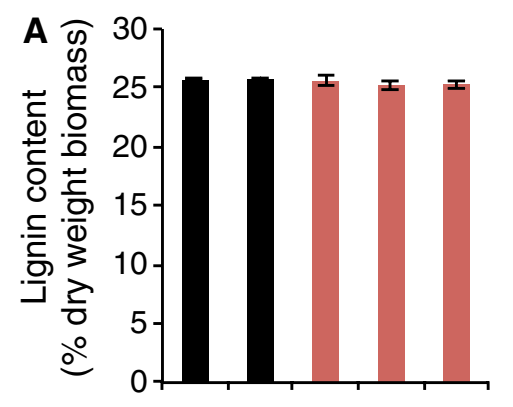

B

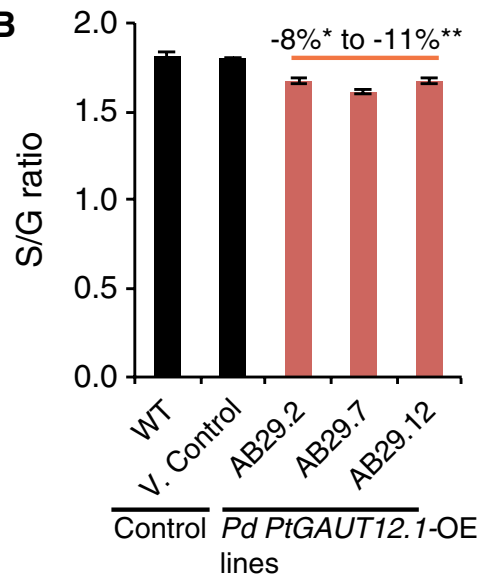

Fig. 5 Total lignin content and S/G ratio of P. deltoides PtGAUT12-OE lines compared to controls. A Total lignin content and $\mathbf{B} S / G$ ratio of P. deltoides WT, vector control, and PtGAUT12.1-OE lines. Data are mean \pm SE. $n=25$ for WT, $n=120$ for vector control (eight different insertion vector control lines, each with $n=15$, see Additional file 2 for the full dataset), and $n=10-15$ for PtGAUT12.1-OE lines, ${ }^{*} P<0.05,{ }^{* *} P<0.001$. Statistical analysis was by one-way analysis of variance (ANOVA) followed by Tukey's multiple comparison test using Statistica 5.0

were carried out on these three selected PtGAUT12.1OE lines.

\section{Reduced growth and increased recalcitrance phenotypes are sustained in field-grown PtGAUT12.1-OE plants}

A field trial was carried out to evaluate the stability of both the PtGAUT12.1-OE genetic modification and the associated phenotypes in the field environment. Seven PtGAUT12.1-OE lines (including AB29.2, AB29.7, and $\mathrm{AB} 29.12)$ were grown alongside $\mathrm{WT}$ and vector control plants for 2.8 years in the field. At the end of the field trial, five of the seven PtGAUT12.1-OE lines had 9-55\% smaller stem radial diameter compared to controls (Fig. 7A, B). The PtGAUT12.1-OE lines also had reduced height compared to the controls, based on visual observation. Quantitative RT-PCR analysis (Fig. 7C) again demonstrated the negative correlation between total GAUT12.1 transcript abundance and plant growth. 

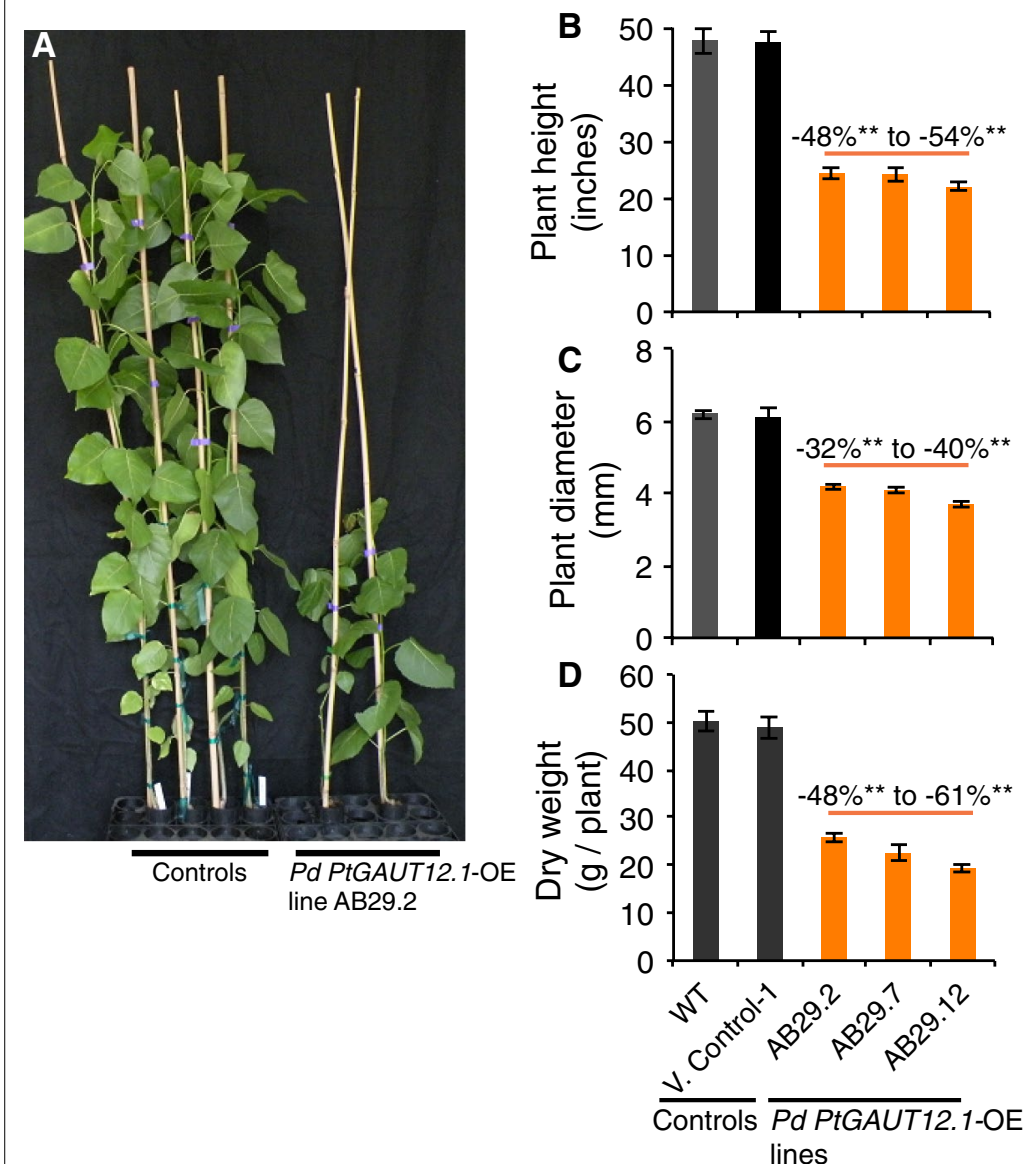
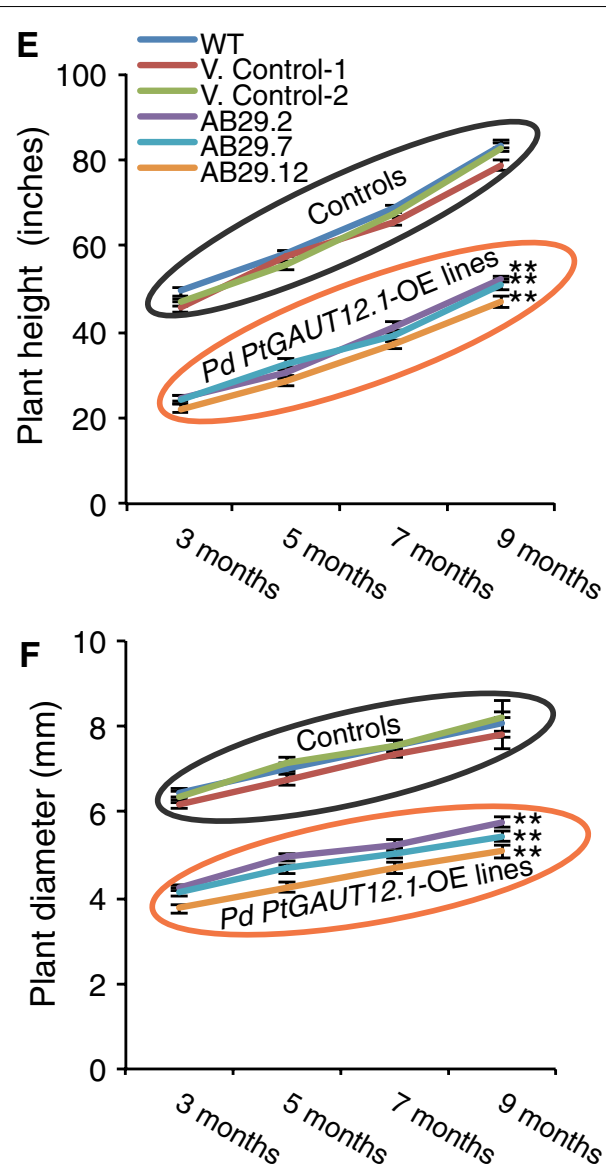

Fig. 6 Growth phenotypes of P. deltoides PtGAUT12.1-OE lines. A Phenotypes of 3-month-old P. deltoides WT (left two plants of shown controls), vector control (right two plants of shown controls), and PtGAUT12.1-OE plants. B Height, C radial growth, and D dry aerial biomass weight of 3-monthold PtGAUT12-OE lines compared to WT and vector control. For height and diameter, $n=25$ for WT, $n=120$ for vector control lines ( $n=10-15$ for each of 8 control lines), and $n=10-15$ for PtGAUT12-OE lines (complete dataset is provided in Additional file 3). For biomass weight, $n=6$. E Height and $\mathbf{F}$ radial growth of greenhouse-grown PtGAUT12-OE and control plants measured over a 9-month growth period $(n=10)$. Error bars represent $\mathrm{SE},{ }^{*} P<0.05,{ }^{* *} P<0.001$

Biomass samples harvested from the field-grown trees were also assessed for recalcitrance characteristics. Glucose, xylose, and total sugar release were all significantly reduced by $10-16,9-17$, and $11-17 \%$, respectively, in the three PtGAUT12.1-OE lines compared to the controls (Fig. 7D-F). Similar to the greenhouse-grown trees, the field-grown PtGAUT12.1-OE lines were not affected in the total lignin content, but were reduced in lignin S/G ratios by $9-14 \%$ compared to the controls (Fig. $7 \mathrm{G}, \mathrm{H}$ ). Taken together, the results confirmed that the genetic manipulation and associated phenotypes were stably maintained in field-grown PtGAUT12.1-OE trees.

\section{PtGAUT12.1 overexpression reduces leaf area and relative water content}

The growth reduction in the PtGAUT12.1-OE lines was also manifested in smaller leaf size (Additional file 5A).
To better evaluate this phenotype, we measured every third successive leaf from the apex down to leaf 25 in both PtGAUT12.1-OE and control lines. Both leaf length and width were significantly reduced in the PtGAUT12.1OE lines (AB29.2, AB29.7, and AB29.12) compared to controls (Additional file 5B, C). We also assessed leaf growth in the PtGAUT12.1-OE and control lines by comparing leaf areas of developing and fully expanded leaves, represented by the 10th and 20th leaf from the apex, respectively. Both developing and fully expanded leaf areas were significantly reduced by $68-74$ and $70-74 \%$, respectively, in all three $\mathrm{OE}$ lines examined compared to the controls (Additional file 5D, E). We then measured the relative water content (RWC), as described previously [5], of leaves from PtGAUT12.1-OE and control plants to determine whether there was a correlation between this parameter and the smaller leaf size in the OE lines. After 

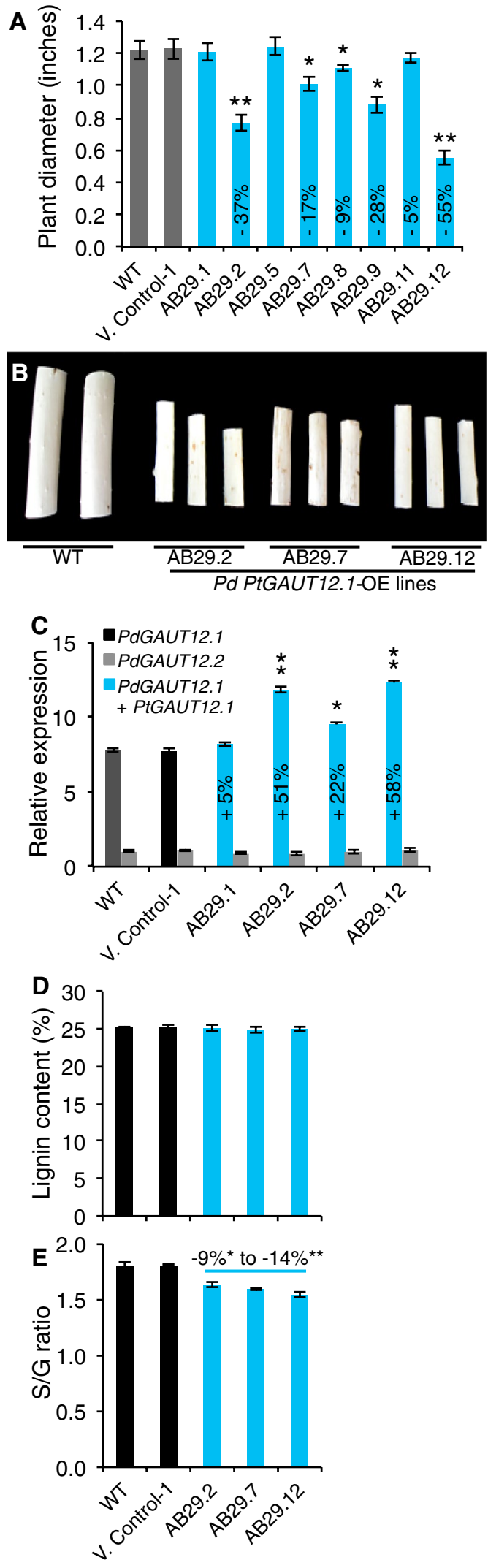
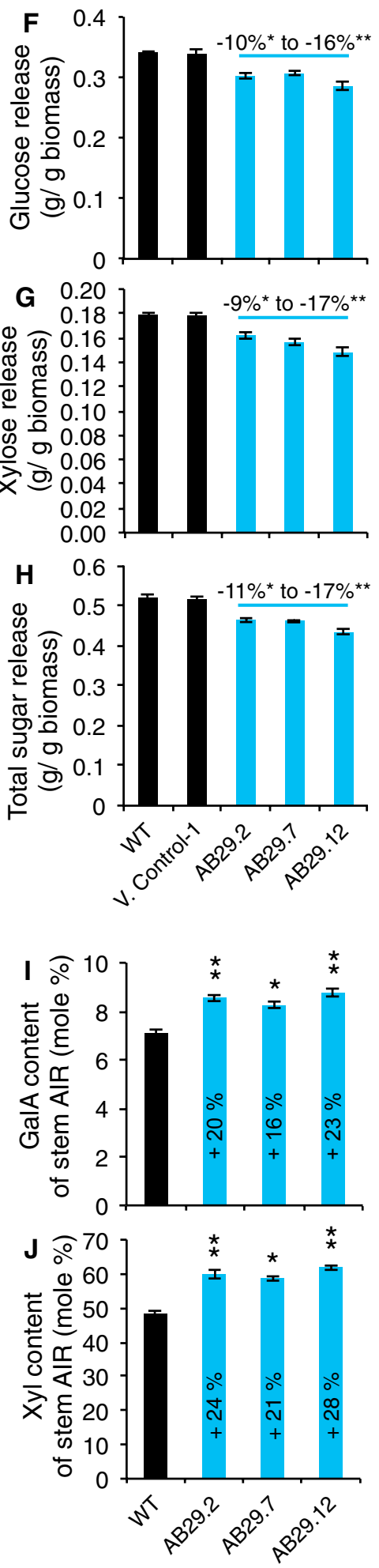
(See figure on previous page.)

Fig. 7 Plant diameter, transcript, lignin, saccharification, and cell wall composition of field-grown P. deltoides control and PtGAUT12.1-OE plants. A Plant diameter and $\mathbf{B}$ debarked stem radial diameter of field-grown PtGAUT12.1-OE plants compared to controls. C Relative abundance of GAUT12.1 transcript determined by quantitative RT-PCR of RNA extracted from stems of 2.8-year-old field-grown trees and normalized to 18S. Expression of PdGAUT12.2 in AB29.7 was set to 1. Each data point represents mean \pm SD of two biological replicates and three technical replicates, $n=4$. D Total lignin content and E S/G ratio of WT, vector control, and PtGAUT12.1-OE lines. F Glucose, G xylose, and $\mathbf{H}$ total sugar release from WT and transgenic lines. I Galacturonic acid (GalA) and $\mathbf{J}$ xylose (Xyl) contents of AlR from stem of 2.8-year-old field-grown WT and PtGAUT12.1-OE plants. $n=4$. Error bars represent SE. ${ }^{*} P<0.05,{ }^{*} P<0.001$

$72 \mathrm{~h}$, the RWC of leaves from PtGAUT12.1-OE lines was 6-12\% lower than in leaves of WT (Additional file 5F). Comparison of relative water content and leaf size in the PtGAUT12.1-OE and PdGAUT12.1-KD lines indicated a positive correlation between these two parameters.

\section{PtGAUT12.1 overexpression decreases the number} of xylem cells and the size of xylem fiber and vessel cells in mature wood tissues

To examine the effect of PtGAUT12.1 overexpression in secondary tissues, WT and PtGAUT12.1-OE 3-monthold plants were analyzed by microscopy of stem sections of the 20th internode from the top of stem. There was a significant $34-41 \%$ decrease in the number of late wood xylem fiber cells per $200 \mathrm{~mm}^{2}$ area in stem sections from the PtGAUT12.1-OE lines compared to WT (Fig. 8A, B, E, F, I, J, M), as well as a significant $19-24 \%$ decrease in the PtGAUT12.1-OE xylem vessel lumen diameter (Fig. 8A, E, I, N). Interestingly, we also observed a 70-91\% increase in xylem fiber cell wall thickness of PtGAUT12.1-OE lines compared to WT (Fig. 8D, H, L, O).

The size of individual wood cells isolated by maceration of debarked bottom stem from 9-month-old plants was also measured (as described previously [5]). Both fiber and vessel cells were smaller in PtGAUT12.1-OE plants compared to WT. Specifically, fiber cells of PtGAUT12.1OE lines were $26-33 \%$ significantly shorter and had $30-40 \%$ reduced diameter compared to WT (Fig. 9A, B). Likewise, the vessel cells of PtGAUT12.1-OE lines were significantly smaller with $18-22 \%$ reduced total length, $15-21 \%$ reduced lumen length, and $19-28 \%$ reduced lumen diameter compared to WT vessel cells (Fig. 9CF). These results indicate that reduced cell number and size in the stem may have led to the reduced plant height and stem diameter in the PtGAUT12.1-OE plants. The results also suggest that overexpression of GAUT12.1 affects both the cell division and expansion in the secondary tissues.

\section{Xylose and galacturonic acid content are increased in PtGAUT12.1-OE walls}

To determine the consequence of PtGAUT12.1 overexpression on the non-cellulosic wall polysaccharides, we analyzed the glycosyl residue composition of wood from the three PtGAUT12.1-OE lines (AB29.2, AB29.7, and AB29.12) along with the WT and vector controls. The goal of these analyses was to test the two propositions regarding GAUT12 enzymatic function. If only xylose was increased in AIR from the GAUT12 overexpression lines, this would support a function of GAUT12 in synthesizing the xylan reducing end sequence. Alternatively, if both xylose and GalA were increased, this would support a function for GAUT12 in synthesizing a pectic glycan required for xylan synthesis.

Wood cell walls were extracted as alcohol insoluble residue (AIR) from the bottom $6 \mathrm{~cm}$ of stems of greenhouse-grown, 9-month-old plants, and analyzed by gas chromatography-mass spectrometry (GC-MS) of trimethylsilyl (TMS) derivatives. The mol\% of two sugars was significantly increased in AIR from PtGAUT12.1OE lines compared to WT, i.e., $14-20 \%$ increased $\mathrm{Xyl}$ and $12-17 \%$ increased GalA (Table 1). There was also a non-significant trend for $19-35 \%$ increased Rha. A significantly greater amount of Xyl (21-28\%) and GalA (16-23\%) was also observed in the glycosyl residue composition of 2.8-year-old, field-grown trees of the same three OE lines compared to WT level (Fig. 7I, J, Additional file 6A), again confirming the stability of the phenotype of the PtGAUT12.1-OE lines in the field environment overtime. The increased $\mathrm{Xyl}$ and GalA content in the PtGAUT12.1-OE walls was accompanied by significantly decreased amounts of galactose (Gal), mannose (Man), and glucose (Glc), compared to controls (Table 1, Additional file 6A). The major effect of PtGAUT12.1 overexpression on the mol\% Xyl and GalA is in agreement with our previous study [5] supporting the role of GAUT12 in xylan and pectin biosynthesis in P. deltoides.

\section{Analyses of fractionated cell walls from transgenic and WT biomass reveal reduced wall extractability, xylan, and HG in the PtGAUT12.1-OE lines}

We reasoned that PtGAUT12.1 overexpression might lead to the increased production of the GAUT12-synthesized polymer in a specific subfraction of wall material and thereby enable its purification and structural characterization. We therefore fractionated AIR samples from 


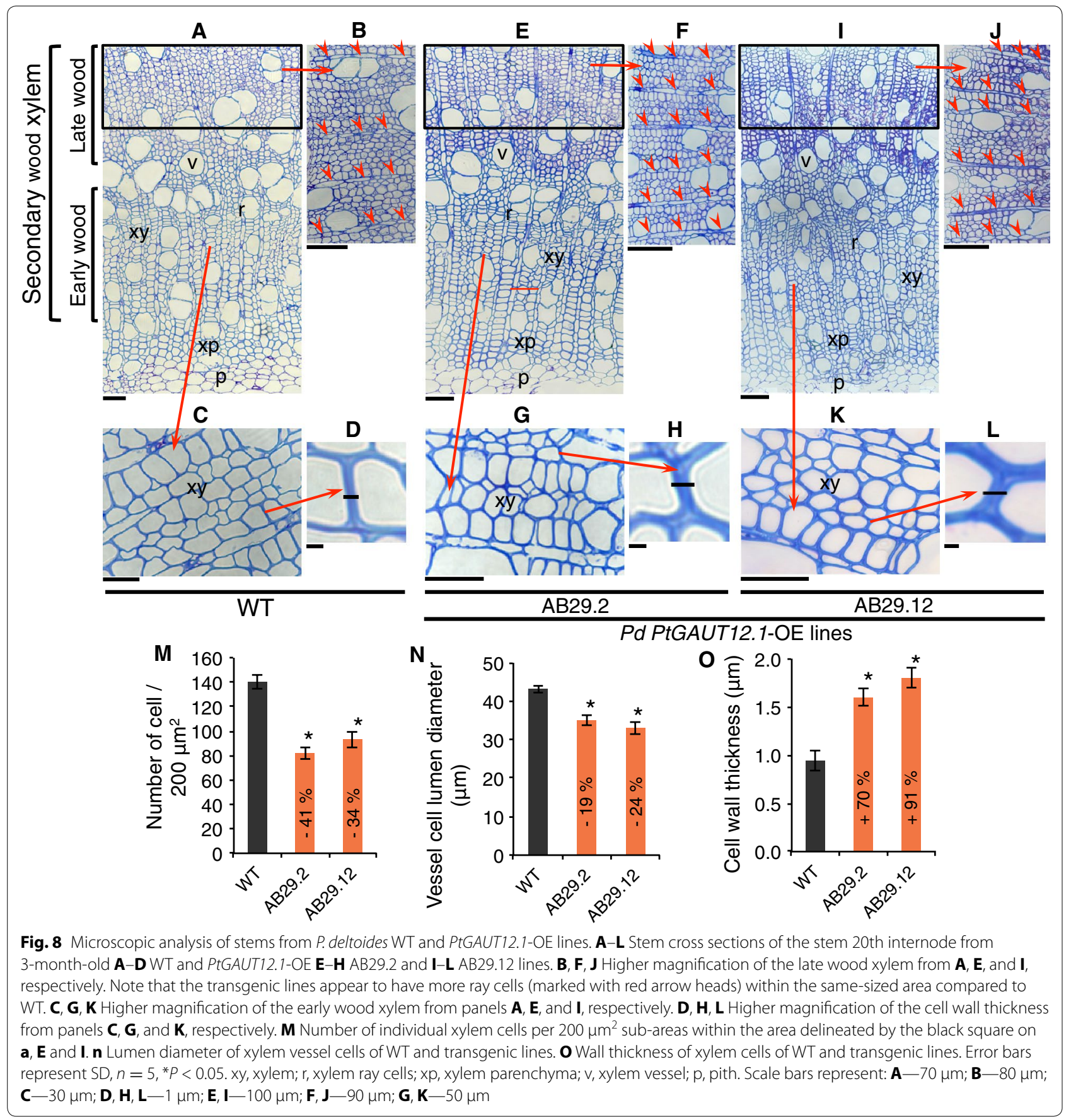

PtGAUT12.1-OE and control lines, from both greenhouse- and field-grown plants, by sequential extraction of AIR using increasingly harsh reagents. This technique yields a series of wall extracts enriched for specific classes of wall polymers $[25,26]$. The wall extracts were analyzed for the amount of wall material recovered, the glycosyl residue composition and linkage, and the presence of specific carbohydrate epitopes via glycome profiling [25].
The goal was to identify a unique polymer(s) produced in the PtGAUT12.1-OE lines.

\section{The amount of extractable cell wall material recovered from PtGAUT12.1-OE lines is reduced compared to WT}

The yields of total AIR recovered from equivalent amounts of PtGAUT12.1-OE and control dry biomass were comparable (Additional file 7A). However, the 

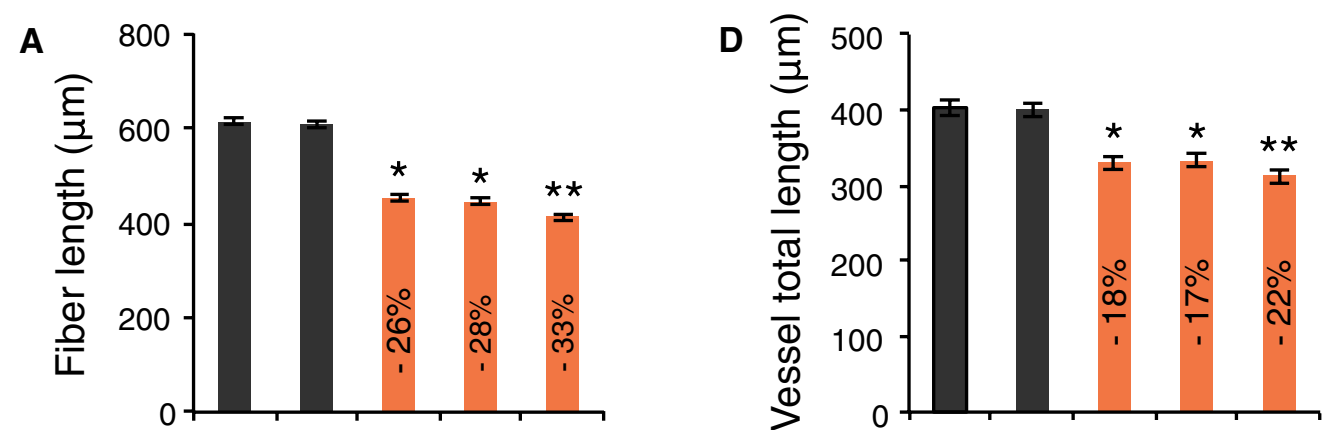

B
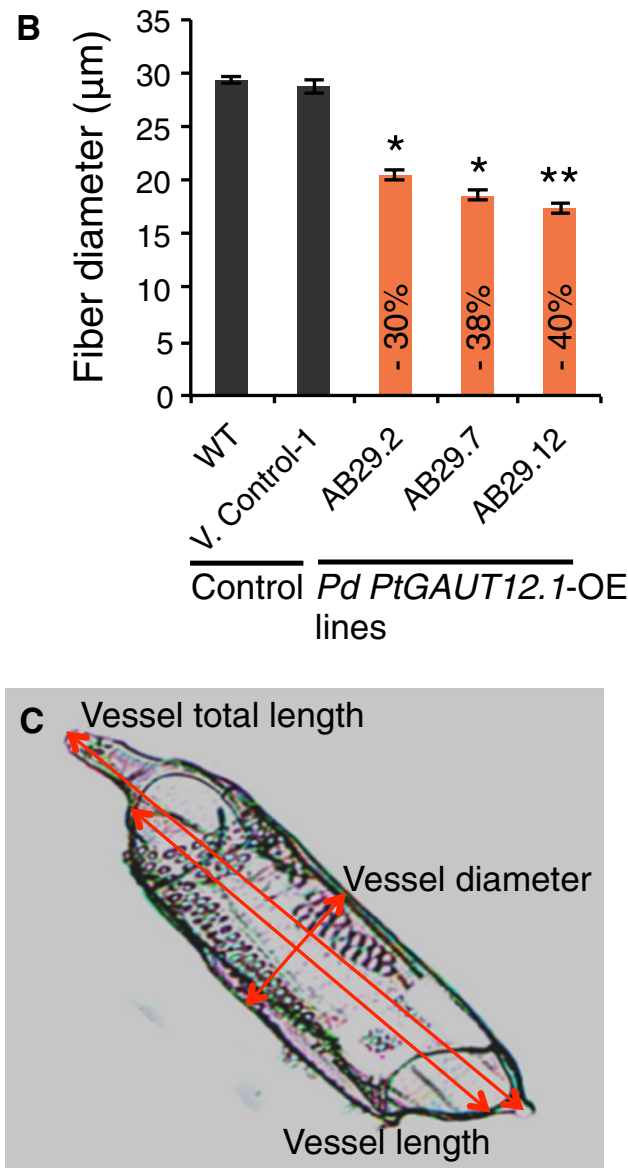

$\mathbf{E}$

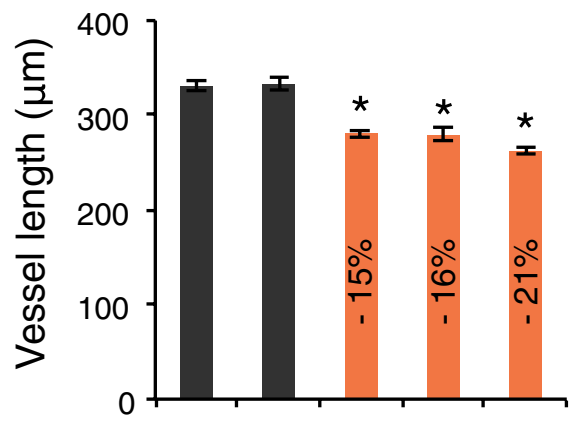

$\mathbf{F}$

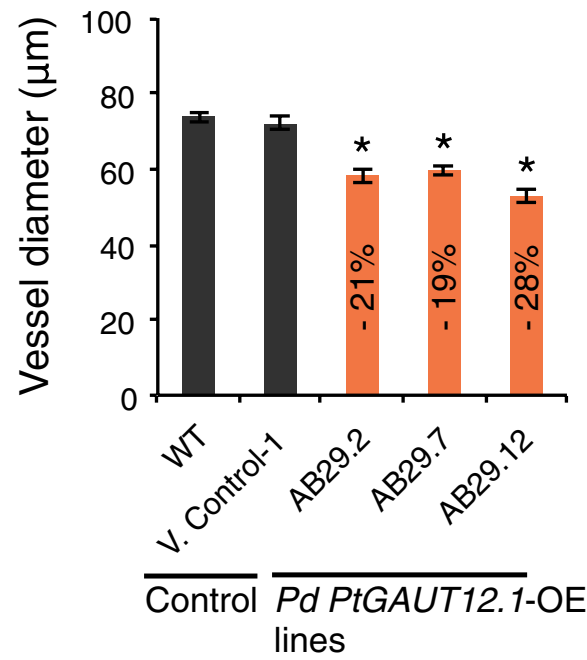

Fig. 9 Xylem fiber and vessel cell size of P. deltoides WT and PtGAUT12.1-OE lines. A, B Xylem fiber length and diameter, respectively. C A vessel cell of PtGAUT12.1-OE line showing the parameters measured. D-F Xylem vessel total vessel length, lumen length, and lumen diameter, respectively. Transgenic values significantly different from wild type, as determined by ANOVA followed by Tukey's multiple comparison test, are denoted with ${ }^{*}(P<0.05)$ or ${ }^{* *}(P<0.001) \cdot n=210$

amounts of wall material recovered in the sequential extracts of the AIR from PtGAUT12.1-OE lines were significantly less than those recovered from the controls. Specifically, the amounts of extractable wall material from the PtGAUT12.1-OE lines were decreased compared to WT by the following amounts in the designated extracts: ammonium oxalate extract (13-25\%), sodium carbonate extract (23-43\%), $1 \mathrm{M} \mathrm{KOH}$ extract (14-22\%),
$4 \mathrm{M} \mathrm{KOH}$ extract (15-22\%), and $4 \mathrm{M} \mathrm{KOH} \mathrm{PC} \mathrm{extract}$ (9-19\%). This resulted in a $9-15 \%$ decreased amount of total recoverable wall material from the combined PtGAUT12.1-OE extracts compared to the controls (Additional file 7B-E, G, H). The only exception was the sodium chlorite extract, for which comparable amounts of extract were recovered from AIR of the PtGAUT12.1$\mathrm{OE}$ and control lines (Additional file 7F). Conversely, 
Table 1 Glycosyl residue composition of alcohol insoluble residue (AIR) from stems of greenhouse-grown, 9-month-old $P$. deltoides WT, vector control, and PtGAUT12.1-OE lines of tetramethylsilane (TMS) derivatives

\begin{tabular}{|c|c|c|c|c|c|c|c|c|c|}
\hline & \multicolumn{9}{|c|}{ Glycosyl residue composition of AIR (mol\% \pm SE) } \\
\hline & Ara & Rha & Fuc & Xyl & GIcA & GalA & Man & Gal & Glc \\
\hline WT & $3.06 \pm 0.06$ & $1.64 \pm 0.06$ & $0.09 \pm 0.006$ & $47.78 \pm 1.21$ & $0.21 \pm 0.008$ & $7.77 \pm 0.21$ & $6.08 \pm 0.25$ & $2.89 \pm 0.14$ & $30.48 \pm 1.20$ \\
\hline V Control-1 & $3.01 \pm 0.08$ & $1.79 \pm 0.04$ & $0.08 \pm 0.004$ & $47.26 \pm 1.32$ & $0.22 \pm 0.006$ & $6.97 \pm 0.14$ & $6.43 \pm 0.28$ & $3.01 \pm 0.21$ & $31.23 \pm 1.32$ \\
\hline AB29.2 & $2.96 \pm 0.10$ & $1.95 \pm 0.06$ & $0.07 \pm 0.007$ & $54.28 \pm 1.01^{*}$ & $0.20 \pm 0.010$ & $8.67 \pm 0.22^{*}$ & $4.11 \pm 0.33^{*}$ & $2.11 \pm 0.16^{*}$ & $25.65 \pm 1.11^{*}$ \\
\hline AB29.7 & $2.88 \pm 0.07$ & $2.01 \pm 0.05$ & $0.10 \pm 0.006$ & $55.64 \pm 1.11^{*}$ & $0.21 \pm 0.005$ & $8.96 \pm 0.23^{*}$ & $4.23 \pm 0.27^{*}$ & $1.97 \pm 0.18^{*}$ & $24.00 \pm 1.21^{*}$ \\
\hline AB29.12 & $2.57 \pm 0.09$ & $2.22 \pm 0.08$ & $0.08 \pm 0.010$ & $57.43 \pm 0.98^{* *}$ & $0.19 \pm 0.007$ & $9.11 \pm 0.24^{* *}$ & $3.15 \pm 0.41^{* *}$ & $1.05 \pm 0.21^{* *}$ & $24.20 \pm 1.25^{*}$ \\
\hline
\end{tabular}

The amounts of sugar are represented as average mol\% of AIR \pm SE of 3 biological and 2 technical replicates $(n=6)$. Italics numbers with stars indicate mutant values that are significantly different from WT at ${ }^{*} P \leq 0.05,{ }^{*} P \leq 0.001$ significant level (one-way ANOVA followed by Tukey's multiple comparison test)

8-15\% more final insoluble pellet was recovered from the PtGAUT12.1-OE AIR compared to the WT lines (Additional file 7I). These results indicate that overexpression of PtGAUT12.1 increases biomass recalcitrance, making it more difficult to extract the wall polymers from the PtGAUT12.1-OE lines than from the control plants. Since only two polymers were increased in abundance in the overexpression line, xylan and HG, the results supported one of the following three hypotheses: (1) increased xylan was inhibiting the ability to extract polymers from the wall, (2) increased HG was affecting wall extractability, or (3) an increased amount of a polymeric structure containing both xylan and HG was increased, restricting wall extractability.

\section{Glycosyl residue composition analysis shows increased GalA and Xyl content in cell wall extracts from PtGAUT12.1-OE versus control lines}

To test the three above hypotheses, the glycosyl residue composition of the different wall extracts was determined. Extraction of AIR using ammonium oxalate and sodium carbonate yields wall extracts enriched in pectic polymers, typified by the abundance of GalA (Table 2, Additional file $6 \mathrm{~B}, \mathrm{C}$ ). For the samples from greenhousegrown plants, the mol\% GalA was significantly increased (by $12-19$ and $21-36 \%$, respectively) in both the ammonium oxalate and sodium carbonate extracts of the PtGAUT12.1-OE lines compared to WT (Table 2). Interestingly, the mol\% Xyl in these wall extracts was also significantly increased (by 21-27 and 17-23\%, respectively) in OE samples compared to the WT (Table 2). In the ammonium oxalate extracts, the greater mol\% GalA and Xyl was accompanied by slightly increased mol\% Rha and Gal in the PtGAUT12.1-OE samples. GalA and Xyl were also increased in the sodium carbonate extracts. Similar trends were found in the field-grown plants (Additional file $6 \mathrm{~B}, \mathrm{C})$. These results suggested that the two pectinenriched extracts of the PtGAUT12.1-OE lines contained increased pectin and xylan content.
Fractionation of the remaining AIR with the alkaline solvents $1 \mathrm{M} \mathrm{KOH}$ and $4 \mathrm{M} \mathrm{KOH}$ extracted cell wall material enriched in hemicellulosic polysaccharides, as indicated by the large amounts of Xyl (Table 2, Additional file $6 \mathrm{D}, \mathrm{E})$. The $1 \mathrm{M}$ and $4 \mathrm{M} \mathrm{KOH}$ extracts of greenhouse-grown PtGAUT12.1-OE lines were significantly increased, compared to WT, respectively, by $14-18$ and 13-27\% mol\% Xyl, and 13-22 and 22-33 mol\% GalA, respectively (Table 2). A $14-15$ and $15-30 \%$ increase in mol\% GlcA was also observed in $1 \mathrm{M}$ and $4 \mathrm{M} \mathrm{KOH}$ extracts, respectively, from PtGAUT12.1-OE lines compared to controls (Table 2). In contrast, the mol\% Man, Gal, and Glc were noticeably decreased in both extracts, as was Rha in the $1 \mathrm{M} \mathrm{KOH}$ extract of PtGAUT12.1-OE lines compared to controls (Table 2). The same trends were observed in samples from field-grown plants (Additional file 6D, E). The results suggest that PtGAUT12.1 overexpression substantially increases the amount of (glucurono)xylan in PtGAUT12.1-OE walls but also affects lesser amounts of pectic polymers present in these fractions.

The insoluble wall material remaining after the $4 \mathrm{M}$ $\mathrm{KOH}$ extraction step was further treated with sodium chlorite to release polymers ostensibly held into the wall by association with lignin (Table 2, Additional file 6F). For the samples from greenhouse-grown plants, Rha, Xyl, and GalA were, respectively, significantly increased by $10-31,15-33$, and $10-30 \%$ in the PtGAUT12.1-OE chlorite extracts, while Man was markedly decreased compared to controls (Table 2). The final post-chlorite (PC) $4 \mathrm{M} \mathrm{KOH}$ extraction step yielded a Xyl-rich extract (Table 2, Additional file 6G). The $4 \mathrm{M} \mathrm{KOH} \mathrm{PC} \mathrm{extracts}$ from greenhouse-grown PtGAUT12.1-OE lines had substantially $13-22 \%$ increased mol\% Xyl, 15-20\% increased GlcA, and 19-33\% increased GalA compared to controls (Table 2) and significantly decreased mol\% Man, Gal, and Glc compared to controls. Lastly, the final insoluble pellets remaining after all extraction steps were analyzed for sugar composition (Table 2, Additional file $6 \mathrm{H}$ ). 
Table 2 Glycosyl residue composition of cell wall fractions from stem of P. deltoides WT, vector control, and PtGAUT12.1OE plants

Glycosyl residue composition of cell wall fractions ( $\mathrm{mol} \% \pm \mathrm{SE}$ )

\begin{tabular}{|c|c|c|c|c|c|c|c|c|c|}
\hline & Ara & Rha & Fuc & Xyl & GlcA & GalA & Man & Gal & Glc \\
\hline \multicolumn{10}{|c|}{ Ammonium oxalate } \\
\hline WT & $19.5 \pm 0.8$ & $5.1 \pm 0.2$ & $0.7 \pm 0.02$ & $8.4 \pm 0.3$ & $0.7 \pm 0.01$ & $23.6 \pm 0.6$ & $11.8 \pm 0.7$ & $4.5 \pm 0.2$ & $25.7 \pm 1.3$ \\
\hline V Control-1 & $18.9 \pm 0.9$ & $4.8 \pm 0.1$ & $0.7 \pm 0.03$ & $8.5 \pm 0.3$ & $0.7 \pm 0.01$ & $23.4 \pm 0.7$ & $12.6 \pm 0.6$ & $4.1 \pm 0.2$ & $26.3 \pm 1.2$ \\
\hline AB29.2 & $17.6 \pm 0.9$ & $5.3 \pm 0.2$ & $0.6 \pm 0.02$ & $10.2 \pm 0.2^{*}$ & $0.7 \pm 0.01$ & $26.5 \pm 0.8^{*}$ & $11.9 \pm 0.4$ & $5.1 \pm 0.1^{*}$ & $22.5 \pm 1.1^{*}$ \\
\hline AB29.7 & $18.1 \pm 0.5$ & $5.6 \pm 0.1^{*}$ & $0.6 \pm 0.02$ & $10.5 \pm 0.3^{*}$ & $0.6 \pm 0.01$ & $27.4 \pm 0.9^{*}$ & $12.1 \pm 0.6$ & $4.8 \pm 0.2$ & $20.2 \pm 0.9^{*}$ \\
\hline AB29.12 & $17.9 \pm 0.6$ & $5.4 \pm 0.2$ & $0.7 \pm 0.03$ & $10.7 \pm 0.4^{* *}$ & $0.7 \pm 0.01$ & $28.1 \pm 0.5^{*}$ & $12.2 \pm 0.5$ & $4.7 \pm 0.1$ & $19.6 \pm 1.1^{*}$ \\
\hline \multicolumn{10}{|c|}{ Sodium carbonate } \\
\hline WT & $11.3 \pm 0.6$ & $5.8 \pm 0.2$ & $0.3 \pm 0.03$ & $22.4 \pm 0.5$ & $1.6 \pm 0.06$ & $18.9 \pm 0.5$ & $15.1 \pm 0.6$ & $6.8 \pm 0.2$ & $17.8 \pm 0.8$ \\
\hline V Control-1 & $11.4 \pm 0.5$ & $6.0 \pm 0.3$ & $0.2 \pm 0.03$ & $22.3 \pm 0.5$ & $1.3 \pm 0.05$ & $18.7 \pm 0.6$ & $15.3 \pm 0.5$ & $6.5 \pm 0.3$ & $18.3 \pm 0.7$ \\
\hline AB29.2 & $8.3 \pm 0.5^{*}$ & $5.1 \pm 0.3$ & $0.4 \pm 0.04$ & $26.3 \pm 0.3^{*}$ & $2.3 \pm 0.06$ & $22.9 \pm 0.4^{*}$ & $14.6 \pm 0.4$ & $5.2 \pm 0.2^{*}$ & $14.9 \pm 0.6^{*}$ \\
\hline AB29.7 & $8.1 \pm 0.4^{*}$ & $5.5 \pm 0.2$ & $0.3 \pm 0.03$ & $26.9 \pm 0.4^{*}$ & $2.4 \pm 0.05$ & $23.6 \pm 0.7^{*}$ & $14.1 \pm 0.6$ & $4.9 \pm 0.2^{*}$ & $14.2 \pm 0.7^{*}$ \\
\hline AB29.12 & $7.8 \pm 0.6^{*}$ & $5.4 \pm 0.3$ & $0.5 \pm 0.02$ & $27.6 \pm 0.6^{*}$ & $2.5 \pm 0.06$ & $25.7 \pm 0.6^{*}$ & $13.5 \pm 0.5^{*}$ & $4.7 \pm 0.3^{*}$ & $12.3 \pm 0.8^{*}$ \\
\hline \multicolumn{10}{|l|}{$1 \mathrm{M} \mathrm{KOH}$} \\
\hline WT & $0.3 \pm 0.01$ & $3.7 \pm 0.1$ & $0.2 \pm 0.01$ & $69.9 \pm 1.3$ & $8.7 \pm 0.2$ & $3.2 \pm 0.1$ & $5.9 \pm 0.2$ & $1.4 \pm 0.08$ & $6.7 \pm 0.2$ \\
\hline V Control-1 & $0.2 \pm 0.01$ & $3.5 \pm 0.1$ & $0.2 \pm 0.01$ & $70.0 \pm 1.5$ & $8.7 \pm 0.3$ & $3.1 \pm 0.1$ & $6.0 \pm 0.3$ & $1.6 \pm 0.05$ & $6.7 \pm 0.4$ \\
\hline AB29.2 & $0.3 \pm 0.02$ & $1.1 \pm 0.1^{*}$ & $0.3 \pm 0.01$ & $79.8 \pm 2.1^{*}$ & $9.9 \pm 0.1^{*}$ & $3.6 \pm 0.05^{*}$ & $2.3 \pm 0.3^{*}$ & $0.9 \pm 0.06^{* *}$ & $1.8 \pm 0.3^{* *}$ \\
\hline AB29.7 & $0.2 \pm 0.01$ & $1.2 \pm 0.3^{*}$ & $0.2 \pm 0.01$ & $81.1 \pm 2.7^{*}$ & $9.9 \pm 0.2^{*}$ & $3.7 \pm 0.06^{*}$ & $1.1 \pm 0.2^{*}$ & $0.6 \pm 0.09^{* *}$ & $2.0 \pm 0.4^{* *}$ \\
\hline AB29.12 & $0.2 \pm 0.01$ & $0.7 \pm 0.3^{*}$ & $0.1 \pm 0.01$ & $82.2 \pm 2.2^{* *}$ & $10.0 \pm 0.2^{*}$ & $3.9 \pm 0.06^{* *}$ & $1.2 \pm 0.4^{*}$ & $0.2 \pm 0.06^{* *}$ & $1.5 \pm 0.5^{* *}$ \\
\hline \multicolumn{10}{|l|}{$4 \mathrm{M} \mathrm{KOH}$} \\
\hline WT & $2.1 \pm 0.09$ & $3.2 \pm 0.1$ & $0.3 \pm 0.02$ & $39.1 \pm 1.4$ & $4.7 \pm 0.2$ & $0.9 \pm 0.03$ & $10.8 \pm 0.5$ & $6.6 \pm 0.2$ & $32.3 \pm 1.6$ \\
\hline V Control-1 & $2.1 \pm 0.03$ & $3.1 \pm 0.1$ & $0.4 \pm 0.02$ & $39.0 \pm 1.1$ & $4.8 \pm 0.1$ & $0.9 \pm 0.03$ & $11.0 \pm 0.3$ & $6.9 \pm 0.3$ & $31.8 \pm 1.7$ \\
\hline AB29.2 & $2.2 \pm 0.06$ & $3.6 \pm 0.08$ & $0.5 \pm 0.02$ & $46.7 \pm 1.3^{*}$ & $5.7 \pm 0.2^{*}$ & $1.2 \pm 0.05^{*}$ & $10.5 \pm 0.6^{* *}$ & $4.9 \pm 0.4$ & $24.7 \pm 1.5^{*}$ \\
\hline AB29.7 & $1.8 \pm 0.05$ & $3.4 \pm 0.1$ & $0.4 \pm 0.01$ & $44.3 \pm 1.1^{*}$ & $5.4 \pm 0.1^{*}$ & $1.1 \pm 0.03^{*}$ & $9.6 \pm 0.3^{*}$ & $5.8 \pm 0.6^{* *}$ & $28.2 \pm 1.4^{*}$ \\
\hline AB29.12 & $2.0 \pm 0.06$ & $3.8 \pm 0.09^{*}$ & $0.3 \pm 0.01$ & $49.8 \pm 1.5^{* *}$ & $6.1 \pm 0.2^{*}$ & $1.2 \pm 0.03^{* *}$ & $8.7 \pm 0.5^{* *}$ & $4.8 \pm 0.3^{*}$ & $23.3 \pm 1.6^{* *}$ \\
\hline \multicolumn{10}{|c|}{ Sodium chlorite } \\
\hline WT & $6.7 \pm 0.3$ & $3.9 \pm 0.2$ & $0.1 \pm 0.01$ & $11.6 \pm 0.4$ & $0.9 \pm 0.03$ & $7.8 \pm 0.2$ & $2.0 \pm 0.04$ & $11.7 \pm 0.3$ & $55.4 \pm 1.5$ \\
\hline V Control-1 & $6.9 \pm 0.4$ & $4.1 \pm 0.2$ & $0.2 \pm 0.01$ & $11.4 \pm 0.6$ & $0.8 \pm 0.04$ & $6.9 \pm 0.2$ & $1.9 \pm 0.06$ & $11.8 \pm 0.4$ & $56.0 \pm 1.4$ \\
\hline AB29.2 & $5.8 \pm 0.4^{*}$ & $5.1 \pm 0.1^{*}$ & - & $14.5 \pm 0.4^{*}$ & $1.1 \pm 0.03$ & $8.9 \pm 0.2^{*}$ & $1.7 \pm 0.08^{*}$ & $11.6 \pm 0.5$ & $51.3 \pm 1.1$ \\
\hline AB29.7 & $6.1 \pm 0.2^{*}$ & $4.3 \pm 0.1^{*}$ & - & $13.3 \pm 0.3^{*}$ & $1.0 \pm 0.02$ & $8.6 \pm 0.1^{*}$ & $1.5 \pm 0.09^{*}$ & $12.3 \pm 0.4$ & $52.9 \pm 1.3$ \\
\hline AB29.12 & $6.2 \pm 0.3^{*}$ & $5.0 \pm 0.2^{*}$ & - & $15.4 \pm 0.5^{* *}$ & $1.3 \pm 0.05$ & $10.1 \pm 0.3^{*}$ & $1.4 \pm 0.1^{* *}$ & $11.1 \pm 0.6$ & $49.5 \pm 0.9^{*}$ \\
\hline \multicolumn{10}{|l|}{$4 \mathrm{M} \mathrm{KOH} P C$} \\
\hline WT & $0.9 \pm 0.03$ & $2.2 \pm 0.06$ & 0 & $66.4 \pm 1.9$ & $8.2 \pm 0.3$ & $2.1 \pm 0.09$ & $6.0 \pm 0.2$ & $4.7 \pm 0.3$ & $9.5 \pm 0.6$ \\
\hline V Control-1 & $0.9 \pm 0.05$ & $2.3 \pm 0.07$ & 0 & $66.0 \pm 2.6$ & $8.1 \pm 0.4$ & $2.0 \pm 0.10$ & $5.9 \pm 0.3$ & $5.0 \pm 0.3$ & $9.8 \pm 0.3$ \\
\hline AB29.2 & $1.0 \pm 0.03$ & $2.5 \pm 0.06^{*}$ & 0 & $78.3 \pm 1.3^{*}$ & $9.6 \pm 0.3^{*}$ & $2.7 \pm 0.04^{*}$ & $1.9 \pm 0.3^{* *}$ & $1.3 \pm 0.2^{*}$ & $2.7 \pm 0.3^{* *}$ \\
\hline AB29.7 & $0.9 \pm 0.04$ & $2.4 \pm 0.08^{*}$ & 0 & $75.1 \pm 1.2^{*}$ & $9.4 \pm 0.2^{*}$ & $2.5 \pm 0.05^{*}$ & $3.5 \pm 0.2^{*}$ & $1.6 \pm 0.3^{* *}$ & $4.6 \pm 0.4^{*}$ \\
\hline AB29.12 & $0.6 \pm 0.06$ & $2.3 \pm 0.07$ & 0 & $80.8 \pm 1.5^{* *}$ & $9.8 \pm 0.4^{* *}$ & $2.8 \pm 0.07^{* *}$ & $1.0 \pm 0.4^{* *}$ & $0.8 \pm 0.2^{* *}$ & $1.9 \pm 0.2^{* *}$ \\
\hline \multicolumn{10}{|l|}{ Insoluble } \\
\hline $\mathrm{WT}$ & $3.9 \pm 0.3$ & $2.8 \pm 0.1$ & $0.2 \pm 0.02$ & $14.9 \pm 0.3$ & $0.2 \pm 0.03$ & $3.0 \pm 0.4$ & $9.5 \pm 0.3$ & $5.6 \pm 0.1$ & $59.9 \pm 0.9$ \\
\hline V Control-1 & $4.0 \pm 0.1$ & $3.1 \pm 0.1$ & $0.3 \pm 0.03$ & $15.3 \pm 0.2$ & $0.2 \pm 0.04$ & $2.9 \pm 0.6$ & $9.9 \pm 0.2$ & $5.4 \pm 0.3$ & $58.9 \pm 0.8$ \\
\hline AB29.2 & $3.9 \pm 0.3$ & $3.5 \pm 0.1^{* *}$ & $0.2 \pm 0.02$ & $16.0 \pm 0.4^{*}$ & $0.2 \pm 0.02$ & $4.9 \pm 0.3^{* *}$ & $5.9 \pm 0.3^{* *}$ & $1.8 \pm 0.2^{* *}$ & $63.6 \pm 0.7$ \\
\hline AB29.7 & $3.8 \pm 0.3$ & $3.8 \pm 0.09^{* *}$ & $0.3 \pm 0.02$ & $16.4 \pm 0.3^{*}$ & $0.3 \pm 0.01$ & $4.7 \pm 0.5^{* *}$ & $4.7 \pm 0.4^{* *}$ & $1.7 \pm 0.3^{* *}$ & $64.3 \pm 0.6^{*}$ \\
\hline AB29.12 & $3.8 \pm 0.3$ & $4.1 \pm 0.1^{* *}$ & $0.3 \pm 0.03$ & $17.0 \pm 0.5^{*}$ & $0.2 \pm 0.02$ & $5.5 \pm 0.4^{* *}$ & $3.5 \pm 0.4^{* *}$ & $1.4 \pm 0.2^{* *}$ & $64.2 \pm 0.7^{*}$ \\
\hline
\end{tabular}

Alcohol insoluble residue (AIR) was sequentially extracted using ammonium oxalate, sodium carbonate, $1 \mathrm{M} \mathrm{KOH}, 4 \mathrm{M} \mathrm{KOH}$, sodium chlorite (chlorite), and $4 \mathrm{M} \mathrm{KOH}$ post-chlorite (PC). Each fraction was then analyzed by GC-MS of tetramethylsilane (TMS) derivatives. The amounts of sugar are average mol\% of wall extracts \pm SE of 3 biological and 2 technical replicates $(n=6)$. Italics numbers with stars are mutant values that are significantly different from WT at ${ }^{*} P \leq 0.05,{ }^{* *} P \leq 0.001$ significant level (one-way ANOVA followed by Tukey's multiple comparison test). A dash indicates that the sugar level was below detection limits 
The greatest increase in the greenhouse PtGAUT12.1OE samples over WT was $63-85 \%$ increased mol\% GalA, followed by $25-46 \%$ increased Rha, and $7-14 \%$ increased Xyl along with a small 6-7\% increase in mol\% Glc (Table 2). These increases were accompanied by a substantial 38-63\% decrease in mol\% Man and 68-75\% decrease in Gal (Table 2). Similar mol\% increase/decrease values were observed in extracts from field-grown plants, indicating that the results were sustained in the fieldgrown lines (Additional file $6 \mathrm{~F}-\mathrm{H}$ ).

The mol\% sugar composition data provide a facile means to compare the relative abundance of the different monosaccharides in total AIR or AIR extracts isolated from transgenic and control lines. Mass yield data, on the other hand, provide information on the actual amounts of the different sugars present in the cell wall samples [27]. We thus also analyzed the $\mu$ g yield of each sugar per mg AIR for each of the cell wall extracts (Additional file 8). In general, the mol\% and mass yield data showed similar general trends. For example, in both data formats, $\mathrm{Xyl}$ and GalA are the only sugar residues whose amounts increased across all wall fractions, including the insoluble pellets. However, there were some minor exceptions. For example, in the PtGAUT12.1-OE samples compared to WT, there was an increased mol\% of GlcA in both the $1 \mathrm{M} \mathrm{KOH}$ and $4 \mathrm{M} \mathrm{KOH}$ extracts, and an increased mol\% of Rha in the $4 \mathrm{M} \mathrm{KOH} \mathrm{PC} \mathrm{extract.} \mathrm{However,} \mathrm{the}$ mass yield data showed a decrease in the total amount of these sugars per mg AIR due to the reduced amount of the $1 \mathrm{M} \mathrm{KOH}, 4 \mathrm{M} \mathrm{KOH}$, and 4MKOHPC fractions in the PtGAUT12.1-OE samples compared to WT (compare Table 2 and Additional file 8). Overall, the data are consistent with GAUT12.1 having a role in the biosynthesis of HG and xylan in P. deltoides and best support hypothesis 3, i.e., that GAUT12.1 is involved in the synthesis of a polymer containing both xylan and HG.

It is interesting to note that analysis of the final pellets remaining after all extractions of AIR from the WT, PtGAUT12-OE, and PdGAUT12-KD lines revealed, surprisingly, that the final pellets contained a greater amount of GalA than any of the extracts (Additional files 8 , 9). Furthermore, the final pellets from the PtGAUT12$\mathrm{OE}$ lines also had the largest increased amount of GalA compared to any of the extracts. This result suggests that poplar GAUT12.1 may function in the synthesis of an HG-containing structure that is part of a foundational cell wall architecture held tightly in the wall and required for the synthesis of xylan.

\section{Glycosyl linkage analysis of PtGAUT12.1-OE cell wall fractions confirms effects on xylan and pectin}

To confirm whether the increased Xyl and GalA contents were indeed associated with xylan and HG, we compared the glycosyl residue linkages of wall carbohydrates recovered in the ammonium oxalate, sodium carbonate, $1 \mathrm{M}$ $\mathrm{KOH}$ wall extracts, and insoluble pellets from AIR of greenhouse-grown PtGAUT12.1-OE lines AB29.2 and AB29.12 and WT (Table 3, Additional file 10). In the ammonium oxalate extracts (Table 3), the greater GalA content in the PtGAUT12.1-OE lines compared to controls was due to a 3-3.7 mol\% increase in 4-linked GalA $p$ and a $0.8 \mathrm{~mol} \%$ increase in terminal-GalA $p$. The 2-linked Rhap, a constituent of the RG-I backbone, was increased $0.2-0.3 \mathrm{~mol} \%$ in these same extracts. The results confirm a higher accumulation of HG accompanied by a smaller increase in RG-I in the cell wall upon PtGAUT12.1 overexpression. Likewise, the increased amount of xylan in the PtGAUT12.1-OE ammonium oxalate extracts was confirmed by the 1.1-1.4 mol\% increased 4-linked Xyl $p$ compared to WT in these samples.

The sodium carbonate extracts from PtGAUT12.1OE had increased mol\% amounts of 4-GalAp (3.8-4.7), 2-GalA $p(0.3-0.4)$, and terminal-GalA $p$ (1.1-1.3) in comparison to controls (Table 3). Although the sugar composition of this wall fraction did not indicate increased amount of Rha (Table 2), the 2-linked Rha $p$ and 2,4-Rhap were increased $0.2-0.4$ and $0.2-0.3 \mathrm{~mol} \%$, respectively, in PtGAUT12.1-OE samples (Table 3). Similarly, we observed 3.6-4.1 increased mol\% 4-Xyl $p$, as well as 0.30.4 terminal-Xyl $p, 0.2-0.32,4-\mathrm{Xyl} p$, and 0.9-1.1 t-GlcA $p$ mol\% increases in the sodium carbonate extracts of PtGAUT12.1-OE compared to WT (Table 3). These data again are consistent with greater quantities of HG and xylan due to the PtGAUT12.1 overexpression.

Following a similar trend as observed in the ammonium oxalate and sodium carbonate AIR extracts, the PtGAUT12.1-OE $1 \mathrm{M} \mathrm{KOH}$ extracts were increased in sugar linkages characteristic of xylan and HG (Table 3). Compared to WT, the PtGAUT12.1-OE samples had 8.7-11.6 mol\% increased for 4-Xyl $p, 0.1 \mathrm{~mol} \%$ increased $\mathrm{t}-\mathrm{Xyl} p$, and $0.8-1.1 \mathrm{~mol} \%$ increased $\mathrm{t}-\mathrm{GlcA} p$, suggesting increased amounts of (glucurono)xylan. This was accompanied by a $0.1-0.3 \mathrm{~mol} \%$ increased 2-GalA $p$ and 0.4-0.6 mol\% increased 3-Rhap in the PtGAUT12.1OE samples compared to WT, suggesting a concurrent increase of the xylan reducing end sequence. The PtGAUT12.1-OE $1 \mathrm{M} \mathrm{KOH}$ extracts also had 3.2$3.4 \mathrm{~mol} \%$ increased $4-\mathrm{GalA} p$ and at least $1.1-1.3 \mathrm{~mol} \%$ $\mathrm{t}$-GalA $p$, consistent with an increased amount of HG.

Interestingly, glycosyl linkage analysis of the final insoluble pellets from the WT and PtGAUT12-OE lines identified fewer types of sugar linkages (Additional file 10) than in the soluble wall extracts (Table 3). Only seven glycosyl linkages were increased in the insoluble pellets of the PtGAUT12.1-OE lines compared to WT. Sugar linkages associated with pectin HG and RG-I backbones, 
Table 3 Glycosyl linkage analysis of cell wall fractions from stems of $P$. deltoides WT and PtGAUT12.1-OE lines

\begin{tabular}{|c|c|c|c|c|c|c|c|c|c|}
\hline & Amn & Im oxalat & able & Sodi & arbonate & & $1 \mathrm{M}$ & oluble & \\
\hline & WT & AB29.2 & $A B 29.12$ & WT & $A B 29.2$ & $A B 29.12$ & WT & $A B 29.2$ & AB29.12 \\
\hline t-Araf & 3.6 & 3.7 & 3.7 & 3.5 & 3.1 & 2.5 & 0.2 & 0.2 & 0.2 \\
\hline t-Arap & 0.2 & 0.2 & 0.2 & 0.2 & 0.2 & 0.2 & 0.1 & 0.1 & 0.1 \\
\hline 3-Araf & 0.5 & 0.7 & 0.8 & 0.5 & 0.4 & 0.4 & - & - & - \\
\hline 4-Arap or 5-Araf & 3.2 & 1.9 & 1.8 & 2.1 & 1.0 & 1.1 & 0.2 & 0.1 & 1.1 \\
\hline 3,4-Arap or 3,5-Araf & 1.5 & - & 1.5 & - & - & - & - & - & - \\
\hline t-Rhap & 0.9 & 0.9 & 0.9 & 1.9 & 1.1 & 1.3 & 0.2 & 0.2 & 0.2 \\
\hline 2-Rhap & 2.2 & 2.4 & 2.5 & 2.9 & 3.1 & 3.3 & 0.7 & 0.4 & 1.1 \\
\hline 3-Rhap & - & - & - & - & - & - & 1.6 & 2.0 & 2.2 \\
\hline 2,4-Rhap & 1.1 & 0.9 & 1.3 & 1.1 & 1.3 & 1.4 & 0.5 & 0.3 & 0.2 \\
\hline t-Fucp & 0.3 & 0.5 & 0.3 & 0.3 & 0.4 & 0.8 & 0.2 & 0.3 & 0.2 \\
\hline t-Xylp & 0.4 & 0.6 & 0.7 & 1.5 & 1.8 & 1.9 & 0.8 & 0.9 & 0.9 \\
\hline 4-Xylp & 6.5 & 7.9 & 7.6 & 20.1 & 23.7 & 24.2 & 57.8 & 66.5 & 69.4 \\
\hline 2,4-Xylp & 0.9 & 1.0 & 1.1 & 1.5 & 1.7 & 1.8 & 8.8 & 5.4 & 3.5 \\
\hline t-Manp & 1.2 & 1.7 & 1.6 & 1.8 & 1.4 & 1.0 & - & - & - \\
\hline 4-Manp & 17.1 & 18.5 & 17.2 & 19.6 & 18.8 & 17.3 & 6.4 & 4.7 & 2.9 \\
\hline 4,6-Manp & 0.7 & - & 0.3 & - & - & - & - & - & - \\
\hline $\mathrm{t}-\mathrm{Glc} A p$ & - & - & - & 1.8 & 2.7 & 2.9 & 7.5 & 8.3 & 8.6 \\
\hline 2-GlcAp & 0.1 & 0.1 & 0.1 & 0.2 & 0.3 & 0.4 & - & - & - \\
\hline t-GalAp & 1.1 & 1.9 & 1.9 & 2.0 & 3.1 & 3.3 & - & 1.1 & 1.3 \\
\hline 2-GalAp & - & - & - & 0.9 & 1.2 & 1.3 & 1.8 & 1.9 & 2.1 \\
\hline 4-GalAp & 23.8 & 26.8 & 27.5 & 16.1 & 19.9 & 20.8 & 1.5 & 4.7 & 4.9 \\
\hline 3,4-GalAp & - & - & - & 0.4 & 0.7 & 0.8 & - & - & - \\
\hline 2,4-GalAp & 0.1 & 0.3 & 0.4 & 0.1 & - & - & - & - & - \\
\hline t-Galp & 2.9 & 2.6 & 2.8 & 3.2 & 2.1 & 2.0 & 1.2 & 0.3 & 0.1 \\
\hline 2-Galp & 0.4 & - & 0.4 & 0.6 & - & - & - & - & - \\
\hline 4-Galp & - & - & - & - & - & - & - & - & - \\
\hline 6-Galp & 0.4 & 0.7 & 0.4 & - & - & - & 0.5 & 0.6 & 0.2 \\
\hline 3,4-Galp & - & 1.7 & 0.4 & 0.3 & - & - & - & - & - \\
\hline 2,4-Galp & 0.2 & 0.2 & 0.2 & 0.2 & - & - & - & - & - \\
\hline $\mathrm{t}-\mathrm{Gl} c p$ & 3.3 & 3.6 & 3.9 & 3.6 & 1.9 & 2.5 & - & - & - \\
\hline 2-Glcp & 0.3 & 0.1 & 0.2 & 0.1 & 0.2 & 0.1 & - & - & - \\
\hline 4-Glcp & 26.7 & 21.1 & 20.0 & 13.5 & 9.9 & 8.7 & 9.8 & 2.0 & 0.8 \\
\hline 4,6-Glcp & 0.4 & - & 0.6 & - & - & - & - & - & - \\
\hline
\end{tabular}

All numbers are mol percentages. A dash designates sugar level below detection limits. Glycosyl linkage analysis data were provided by CCRC Analytical Services

4-GalAp, t-GalAp, and 2,4-Rhap, were increased by up to $0.5,0.3$, and $0.2 \mathrm{~mol} \%$, and sugar linkages associated with xylan, 4-Xyl $p$, t-GlcA $p$, were increased by 0.2 and $0.1 \mathrm{~mol} \%$, respectively, in PtGAUT12.1-OE insoluble pellets compared to WT. The only other glycosyl linkages increased in the final pellets of PtGAUT12.1OE lines compared to WT were 4-Man $p$ and t-Glcp which were increased by 5.1 and $0.4 \mathrm{~mol} \%$, respectively. Intriguingly, analysis of the final pellets remaining in the PdGAUT12.1-KD lines (Additional files 10) also revealed the same limited types of glycosyl linkages, and of those only nine glycosyl linkages were decreased in the insoluble pellets of PdGAUT12.1-KD lines compared to WT. Sugar linkages associated with pectin HG and RG-I backbones, 4-GalAp, t-GalAp, and 2,4-Rhap, were decreased by up to $0.8,0.2$, and $0.2 \mathrm{~mol} \%$, respectively, and sugar linkages associated with xylan, 4-Xyl $p$, $\mathrm{t}$-GlcA $p$, were decreased by 0.3 and $0.2 \mathrm{~mol} \%$, respectively, in the PdGAUT12.1-KD insoluble pellets compared to WT. The other glycosyl linkages decreased in the final pellets of the PtGAUT12.1-OE lines compared to WT were 4-Man $p$, 4,6-Man $p$, t-Glcp, and 3,4-Glcp which were decreased by $3,0.1,0.4$, and $0.1 \mathrm{~mol} \%$, respectively. Taken together, the glycosyl linkage data provide support 
for the hypothesis that poplar GAUT12.1 is involved in the synthesis of an HG-containing glycan that contains an RG-I, RG-I/xylan (see the Arabinoxylan Pectin Arabinogalactan Protein1 (APAP1) proteoglycan structure in [28]) or novel pectin-xylan structure required for the synthesis of (glucurono)xylan synthesis.

\section{Glycome profiling reveals increased binding of specific xylan and $H G$ backbone antibodies in certain wall fractions of PtGAUT12.1-OE compared to control}

To further investigate the types of wall glycans increased in the PtGAUT12.1-OE lines, and antibodies that may recognize these structures, we subjected the sequentially extracted wall fractions from PtGAUT12.1-OE and WT lines to glycome profiling analyses. A set of 155 monoclonal antibodies (mAbs) raised against diverse noncellulosic plant cell wall polysaccharides and reactive to different non-cellulosic glycan epitopes [29] was used to screen the wall extracts in this ELISA-based assay [25], with the goal of obtaining information about the presence and relative abundance of specific epitopes that are characteristic of different types of non-cellulosic polymers in each extract. The binding strength of each $\mathrm{mAb}$ across the different wall extracts and plant lines was visualized as heat maps [25].

The glycome profiling data (Fig. 10) showed both increases and decreases in epitope contents in the PtGAUT12.1-OE cell wall extracts compared to WT. The most consistent changes across multiple extracts were observed for xylan backbone epitopes recognized by the Xylan- 6 and Xylan-7 groups of mAbs [30, 31], 4-O-methyl-GlcA-substituted xylans recognized by the Xylan- 5 mAbs [30], and the epitopes recognized by HG backbone-specific mAbs [32]. Increased binding of mAbs that specifically bind to the 4-O-methyl GlcA side chains of xylans was observed in the oxalate, carbonate, chlorite, and $4 \mathrm{M}$ KOHPC extracts of PtGAUT12.1-OE compared to WT (green boxes, Fig. 10). In addition, increased binding of CCRC-M150, which is specific for GlcAsubstituted xylan [30], was observed in the $4 \mathrm{M} \mathrm{KOHPC}$ extract. No binding of CCRC-M154, a mAb specific for arabinosyl-substituted xylans [31], was observed in any cell wall extract from either OE or WT lines. Decreased binding of xylan backbone-directed mAbs (Xylan-6 and Xylan-7) was observed in the $4 \mathrm{M} \mathrm{KOH}$, chlorite, (orange boxes, Fig. 10) and to a lesser extent, in the $4 \mathrm{M} \mathrm{KOH} \mathrm{PC}$ extracts. For HG polymers, there was increased binding of mAbs directed against the de-methylesterified (HG backbone-1) and methylesterified (HG backbone-2) HG backbones in the oxalate extracts from PtGAUT12.1-OE AIR compared to WT, while in the other wall extracts (carbonate, chlorite, and $4 \mathrm{M} \mathrm{KOHPC)} \mathrm{there} \mathrm{was}$ increased binding of only the de-methylesterified HG backbone-directed mAbs in the OE lines (blue boxes, Fig. 10). In contrast, decreased binding was evident for mAbs in the RG-I/AG and AG-2, -3 , and -4 groups in carbonate extracts and for non-fucosylated xyloglucan groups 3-6 in $4 \mathrm{M} \mathrm{KOH}$ extracts of the PtGAUT12.1-OE lines compared to WT (white boxes, Fig. 10). Overall, the glycome profiling data demonstrate altered wall polymer extractability as a result of GAUT12.1 overexpression in poplar, with a trend towards more recalcitrant biomass from which it becomes harder to extract wall polymers.

\section{Discussion}

We have overexpressed PtGAUT12.1, the higher expresser of the two poplar orthologs of Arabidopsis GAUT12, in P. deltoides. The generated poplar transgenics had 7-49\% increased total GAUT12.1 (both the introduced PtGAUT12.1 transgene and the endogenous PdGAUT12.1) transcript expression level, leading to a $4-12 \%$ reduced saccharification yield for greenhousegrown transgenic biomass. Plant growth was also negatively affected in PtGAUT12.1-OE lines, with $6-54 \%$ reduced plant height, $8-41 \%$ reduced radial stem diameter and, most importantly, $48-61 \%$ reduced dry biomass yield compared to controls. The elevated transcript level, increased recalcitrance, and decreased growth were notably maintained in PtGAUT12.1-OE plants grown for more than 2 years in the field, demonstrating the transgene stability in the environment over time. Most importantly, PtGAUT12.1-OE plants displayed exactly the opposite phenotypes of PdGAUT12.1-KD (knockdown) plants described in our previous research [5], in which downregulation of PdGAUT12.1 by RNA silencing resulted in significantly increased saccharification efficiency and improved growth. Furthermore, the opposing phenotypes of the PtGAUT12.1-OE versus PdGAUT12.1-KD lines extend to almost all parameters measured in this work, including leaf phenotypes, cell size, and extractability, sugar composition, and sugar linkages of the cell wall. Overall, the combined $\mathrm{OE}$ and KD data clearly establish GAUT12.1 as a recalcitranceand growth-associated gene in poplar.

The phenotypes of the PtGAUT12.1-OE plants verified our previous results [5] showing that modifying GAUT12 expression in poplar yields outcomes different than in Arabidopsis. The knocking-out of Arabidopsis GAUT12/ IRX8 resulted in reduced wall xylan content, a collapsed xylem phenotype, and dwarfed ir $x 8$ mutant plants [13, 14], while overexpression of the gene did not alter wall composition or plant growth [15]. We showed previously that PdGAUT12.1 could complement the phenotype of Arabidopsis irx 8 mutants, indicating that it is a functional ortholog of the Arabidopsis GAUT12 [5]. However, silencing of PdGAUT12.1 in poplar, while also causing 


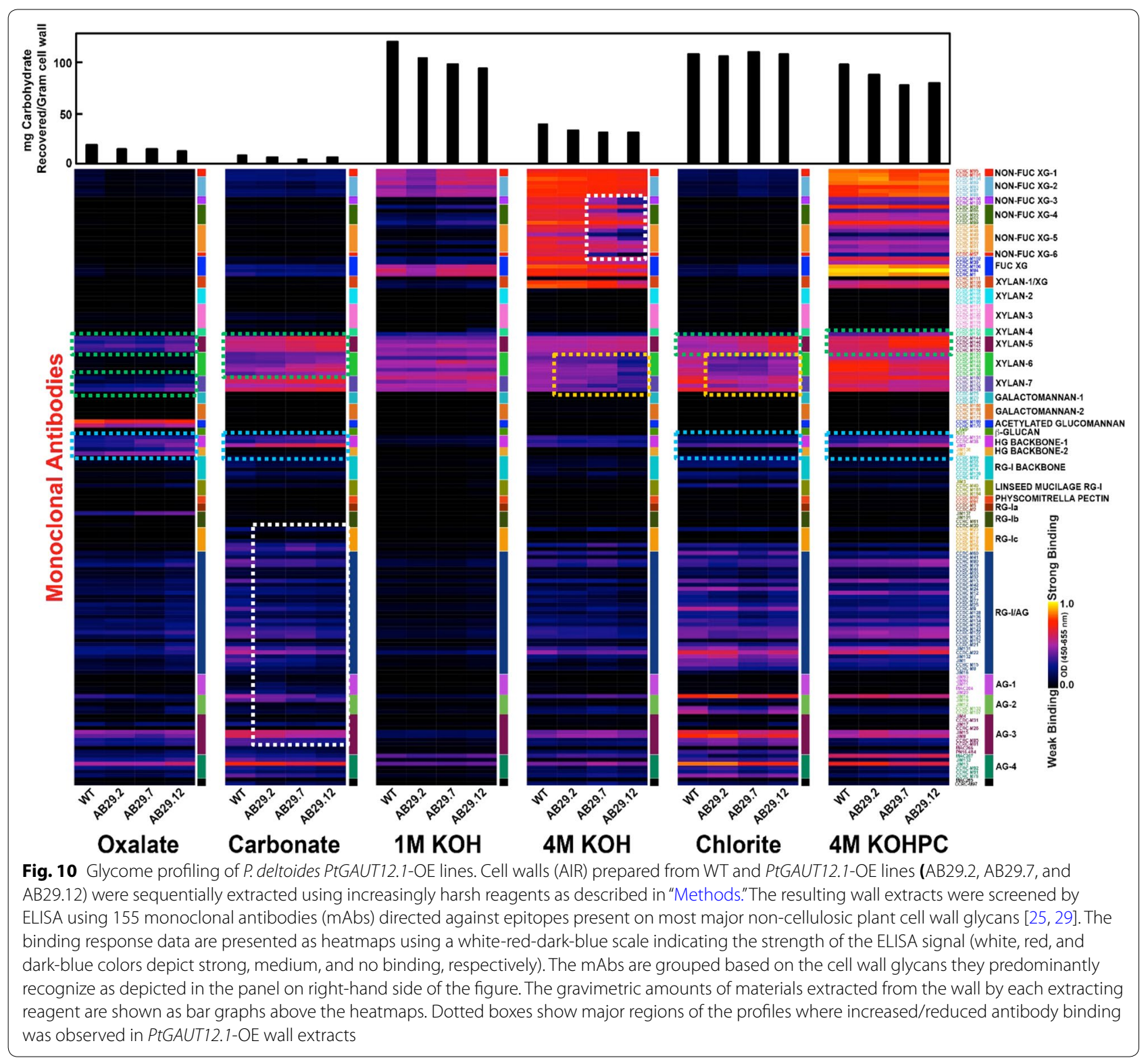

significantly reduced xylan content, resulted in increased saccharification without compromising growth; rather it increased plant growth [5]. The increased xylan content and reduced plant growth of the PtGAUT12.1-OE plants, thus confirmed that the effects of modifying expression of a gene in Arabidopsis does not necessarily translate to woody plants and, as such, highlights the need for phenotypes to be verified across species.

The reduced plant growth and biomass yield of the PtGAUT12.1-OE lines compared to controls may, at least partially, be due to the decreased leaf size, xylem vessel cell size, and relative water content. Since water flow is smaller in smaller radius vessels [33], the reduced cell size of xylem vessel cells could explain the reduced water content and poorer growth of PtGAUT12.1-OE compared to WT. In contrast, PdGAUT12.1-KD lines had the reverse characteristics [5] with increased cell size, water content, and plant growth. Such effects of modified GAUT12.1 expression on cell size, water content, and wall integrity suggest that the mechanism(s) by which modified GAUT12 expression leads to modified cell and plant growth may be complex and multifactorial. Previous studies of GAUT12 have focused heavily on the stem due to the high GAUT12 transcript expression in this tissue. However, weaker expression of GAUT12 has also been reported in the vascular tissues 
of leaves and petioles of Arabidopsis and poplar [5, 11, $14,22,34]$. We show here that weak expression of poplar GAUT12, especially PdGAUT12.1, indeed is detectable in $P$. deltoides developing organs, i.e., apex, young leaves, and young internodes (Fig. 2C, D). This suggests that at least some effects of modifying GAUT12.1 expression may already have taken place in young tissues and may explain the reduction and increase in the overall growth of PtGAUT12.1-OE and PdGAUT12.1-KD plants, respectively, as opposed to phenotypes occurring only in the secondary wall-rich stem. The PtGAUT12.1-OE growth phenotype, however, may also be due to a plantwide effect as a result of the use of A. thaliana Ubiquitin3 promoter to drive the overexpression of PtGAUT12.1 constitutively in $P$. deltoides.

Cell wall sugar composition analyses revealed substantially increased Xyl and GalA content in the total AIR and also in virtually every single wall fraction extracted from AIR of the PtGAUT12.1-OE biomass compared to controls, both from greenhouse- and field-grown plants (Tables 1, 2, Additional files 6, 8). Together with the sugar linkage data (Table 3, Additional file 10), these data indicated increased amounts of xylan and HG as a result of PtGAUT12.1 overexpression, and were consistent with the hypothesis that GAUT12 encodes a GT that synthesizes a wall structure containing, or required for the formation of, both xylan and HG in poplar wood.

Analysis of the sugar composition, linkage, and glycome profiling data of $P$. deltoides PdGAUT12.1-KD (Additional files 9, 10, and in [5]) and PtGAUT12.1-OE (Tables 1, 2, 3, Additional files 8, 10, Fig. 10) biomass revealed several interesting observations. First, both Xyl and GalA contents were increased in all PtGAUT12.1OE wall fractions compared to controls (Table 2, Additional file 8). However, only GalA content was reciprocally decreased in every single wall extract of the PdGAUT12.1-KD biomass (Additional file 9, [5]). Xyl content was indeed reduced in the total AIR and in most of the wall extracts of the PdGAUT12.1-KD biomass, yet it was increased in the first two wall fractions, i.e., the ammonium oxalate and sodium carbonate extracts (Additional files 9, [5]). These results suggest that knockdown of PdGAUT12.1 led to a subfraction of xylan that was more easily extracted from the wall. These changes in GalA content, rather than in Xyl, in all wall fractions suggest that PdGAUT12.1 functions directly in the synthesis of a specific HG glycan required for xylan synthesis, rather than directly in the synthesis of xylan itself.

Secondly, it is noteworthy that a substantial portion of GalA actually resides in the insoluble pellets of both the PdGAUT12.1-KD and PtGAUT12.1-OE cell walls that remain after all the extractions steps (as can be seen in Additional files 8,9 ). In addition, a large percentage of the GalA is also present in the $1 \mathrm{M} \mathrm{KOH}, 4 \mathrm{M} \mathrm{KOH}$, and chlorite fraction. This is contrary to the belief that the bulk of pectin is released in wall fractions extracted with ammonium oxalate, CDTA [16], or sodium carbonate. Rather, these data indicate that a significant portion of pectin is tightly held in the wall. This finding is consistent with the recent solid-state nuclear magnetic resonance (SS-NMR) data showing that a substantial portion of pectin (including HG and RG-I) is in close spatial contact with cellulose in Arabidopsis walls [35-37].

Thirdly, the increase and decrease of the Xyl and GalA content in the PtGAUT12.1-OE and PdGAUT12.1-KD lines, respectively, appeared to be concomitant with trends in the reversed direction of the Man, Gal, and Glc content, i.e., the latter sugars were increased in the KD biomass and decreased in the OE biomass (Table 2, [5], Additional files 8, 9). This was observed both in total AIR and in almost all wall fractions, and was especially obvious in the mass ( $\mu$ g sugar/mg AIR) data. Such trends suggest that the decrease in xylan and HG synthesis by knocking-down PdGAUT12.1 expression was compensated by increased synthesis of wall polymer(s) that contain Man, Gal, and/or Glc, and vice versa in the PtGAUT12.1-OE transgenics. The available sugar linkage data showed mostly similar trends for $\mathrm{t}-\mathrm{Gal} p$, 4-Man $p$, 4,6-Man $p$, and 4-Glcp (Table 3, Additional file 10, [5]), suggesting that the hemicelluloses mannan, including galactomannan and glucomannan, are the likely candidate polymers affected. Indeed, poplar wood contains $\sim 5 \%$ glucomannan, and a glucomannan synthase gene CSLA1/GT2A is known to be highly expressed in poplar during the transition from primary to secondary walls $[6,38,39]$. From the biofuel production perspective, it is conceivable that bioethanol production would benefit from the increased amounts of hexoses Man, Gal, and Glc in the PdGAUT12.1-KD biomass compared to controls [5], in addition to the looser walls and increased wall polymer extractability.

Fourthly, it is interesting to note that the Ara and Rha content were negatively affected by both down- and upregulation of GAUT12.1 expression in the majority of wall fractions of the transgenic biomass (Table 2, Additional files 8, 9, [5]). However, in the total AIR (Table 1, [5]), Ara content was reduced in both KD and OE biomass, while the Rha content of the KD lines was comparable to WT and that of the OE lines was increased compared to WT. The sugar linkage data (Table 3, [5]) showed that $\mathrm{t}$-Araf and 5-Araf sugar linkages were reduced or comparable in the ammonium oxalate and sodium carbonate extracts of both PdGAUT12.1-KD and PtGAUT12.1-OE biomass, suggesting arabinan as the polymer affected in this case. Such trends suggest indirect effects of the transgenesis on the Ara- and Rha-containing wall polysaccharides. 
Fifthly, the glycome profiling data revealed that 4-O-methyl-substituted xylan and de-methylesterified HG backbone epitopes increased simultaneously in the oxalate, carbonate, chlorite, and $4 \mathrm{M} \mathrm{KOHPC}$ PtGAUT12.1-OE wall extracts compared to controls. Although such data are semi-quantitative, it is plausible that the epitopes recognized by these two mAbs groups reside within the same polysaccharide structure, hence facilitating their co-extraction into these wall fractions. The four wall extracts could be investigated further by chromatographic separation coupled with analysis of the fractions by detection of the epitopes using the mAbs in ELISA assays, with the goal of purifying the xylanand HG-containing polymer(s) for further structural characterization.

The impacts of modified GAUT12.1 expression on xylan and HG content led us to hypothesize that
GAUT12.1 is a GalAT that catalyzes the incorporation of GalA residues into one or more of four hypothetical structures (Fig. 11): (1) the xylan reducing end sequence, (2) an HG primer for xylan, (3) an HG in an APAP1-like primer for xylan, and (4) a unique HG glycan not covalently attached to xylan. Here we critically evaluate each possible structure based on the combined sugar composition and linkage data from both the PdGAUT12.1-KD and PtGAUT12.1-OE biomass.

\section{Hypothetical structure 1}

Compromised synthesis of the xylan reducing end sequence would manifest in the KD biomass as a reduction in either total AIR, or in one or more wall fractions, in the amounts of 2-GalA $p$, 3-Rha $p$, and 4-Xyl $p$ of the tetrasaccharide sequence, as well as the 4-Xylp of the xylan backbone, and vice versa in the OE biomass. Such effects

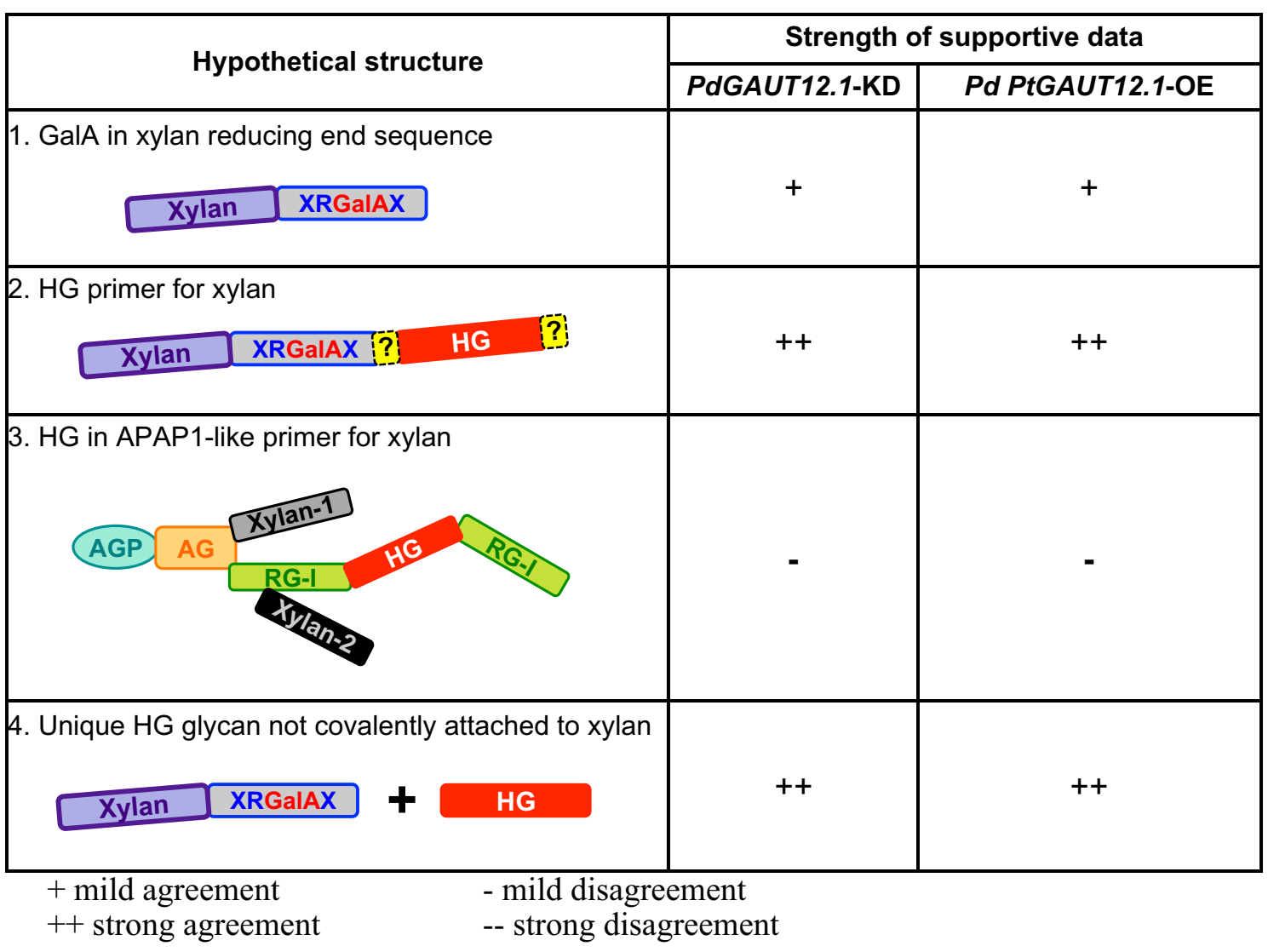

Fig. 11 Hypothetical structures that may be synthesized by poplar GAUT12.1. Depiction of four possible structures that could be synthesized by GAUT12 based on available published data on the chemistry and biochemistry of cell walls and cell wall fractions from GAUT12 mutants and transgenics versus their respective controls. The rectangles depict glycan domains of xylan, the xylan reducing end sequence (XRGalAX), homogalacturonan (HG), arabinogalactan (AG), rhamnogalacturonan I (RG-I), and the two unique xylan regions (Xylan-1, Xylan-2) of the plant proteoglycan Arabinoxylan Pectin Arabinogalactan Protein 1 (APAP1). The oval depicts the arabinogalactan protein (AGP) core of APAP1. The question marks (?) depict hypothetical covalent linkages. Hypothesized function of GAUT12 in structures shown: structure 1-insertion of GalA into the xylan reducing end sequence; structure 2-synthesis of HG covalently connected directly or indirectly to xylan/xylan reducing end sequence; structure 3-synthesis of HG domain in APAP1; structure 4-synthesis of HG associated with xylan synthesis but not covalently bound to the xylan polymer. Strength of supportive published data and data from this work: + mild agreement, ++ strong agreement, - mild disagreement; -- strong disagreement 
were observed in the $1 \mathrm{M} \mathrm{KOH}$ extracts (Table 3, [5]) that were enriched in hemicellulosic polymers, supporting GAUT12.1 function in the synthesis of the xylan reducing end sequence. However, such a GAUT12.1 function could not explain the substantially altered GalA contents observed in every single wall fraction in both the $\mathrm{KD}$ and $\mathrm{OE}$ transgenics, thus casting doubt on the role of GAUT12.1 in the synthesis of the xylan reducing end sequence.

\section{Hypothetical structure 2}

Here we define a xylan structure with an HG primer as a xylan covalently connected to a GAUT12-synthesized HG by yet-to-be-identified glycosyl, base-sensitive, or other linkages. The possibility that GAUT12.1 synthesizes an HG primer for xylan is supported by the concomitant reductions in the amounts of GalA and Xyl in the total AIR and in the $1 \mathrm{M} \mathrm{KOH}, 4 \mathrm{M} \mathrm{KOH}$, chlorite, $4 \mathrm{M} \mathrm{KOHPC}$, and insoluble wall extracts of the KD biomass (and vice versa for the OE biomass; Tables 1, 2, Additional files 8, 9, [5]), suggesting that such an HG primer for xylan might be recovered in these wall fractions. Furthermore, 4-GalA $p$ and 4-Xyl $p$ glycosyl linkages were decreased in the $1 \mathrm{M} \mathrm{KOH}$ and insoluble wall fractions in the $\mathrm{KD}$ biomass and increased in the $\mathrm{OE}$ biomass (Table 3, Additional file 10, [5]).

\section{Hypothetical structure 3}

An APAP1-like structure with a GAUT12.1-synthesized HG glycan would be expected to contain 4-GalA $p$ for the HG glycan, 2-Rha $p$ and/or 2,4-Rhap for the RG-I glycan, and $4-X y l p$ for the xylan glycan. The altered GAUT12.1 expression would have been expected to affect the contents of these sugars and linkages simultaneously in the wall extracts. We did observe such changes for GalA and Xyl (see description for the "Hypothetical structure 2" above), but not for Rha. The expected trend in Rha content was observed only in the chlorite and insoluble wall fractions, i.e., reduced in the PdGAUT12.1-KD and increased in the PtGAUT12.1-OE samples, and not in the other PtGAUT12.1-OE cell wall fractions. Furthermore, there was not a consistent trend for the 2-Rhap and 2,4Rhap linkages in the wall fractions tested (Tables 2, 3, Additional files 8, 9, [5]). Taken together, the results do not support a role for GAUT12.1 in the synthesis of an APAP1-like structure.

\section{Hypothetical structure 4}

The data showed that GalA content and 4-GalAp linkage were affected in all wall extracts for which the sugar composition and linkage data were available (Tables 2, 3 , Additional files 8, 9, 10, [5]). These results could be consistent with a function of GAUT12.1 in the synthesis of a unique HG glycan that is not necessarily covalently attached to xylan, but that still affects xylan synthesis.

Based on a consideration of all the available cell wall sugar composition and linkage data from the PdGAUT12.1-KD and PtGAUT12.1-OE transgenics, the results could support a function for GAUT12 in synthesizing either structures 2 or 4 . However, the recovery of increased $\mathrm{Xyl}$ in the oxalate and carbonate fractions of the PdGAUT12.1-KD lines, while the amount of GalA was decreased in the same fractions [5], leads us to favor hypothetical structure 2 as the polymer synthesized by poplar GAUT12.1, i.e., an HG-containing wall polymer that is covalently linked to xylan. We propose that in the PdGAUT12.1-KD transgenics, such a structure was not produced at the level required for native wall synthesis, resulting in some xylan not being properly connected in the wall. This scenario is supported by the increased Xyl content and much higher Xyl/GalA ratios in the ammonium oxalate and sodium carbonate wall extracts from PdGAUT12.1-KD compared to WT, suggesting that such poorly integrated xylan was released in these wall fractions. We further propose that in the PtGAUT12.1-OE plants, overexpression of PtGAUT12.1 caused increased production of hypothetical structure 2, leading to decreased wall polymer extractability, increased amounts of the insoluble residues remaining after all extractions, increased biomass recalcitrance, and restricted cell and plant growth in the transgenic OE lines. Our data do not show how the HG and xylan are covalently connected in hypothetical structure 2. It is possible that the HG glycan, having been synthesized first, acts as a primer onto which the xylan is synthesized. It is also possible that the HG and xylan glycans are synthesized independently, and subsequently connected together in the secretory pathway. Another alternative is that the HG and xylan are synthesized and secreted into the apoplast independently, and the covalent connection between them occurs in the wall. In the latter two possibilities, the HG glycan of hypothetical structure 2 would serve to anchor the xylan glycan for proper deposition/integration into the wall architecture. Further research is necessary to identify the covalent linkage(s) and the structure/architecture.

Finally, based on the yields of xylan obtained upon extraction of the cell walls with basic solvents, we propose that the covalent connection between the HG and xylan in the GAUT12.1-synthesized structure may be base-sensitive, and thus, is cleaved during the $1 \mathrm{M} \mathrm{KOH}$, $4 \mathrm{M} \mathrm{KOH}$, and $4 \mathrm{M} \mathrm{KOHPC} \mathrm{extraction} \mathrm{steps} \mathrm{resulting}$ in high levels of xylan in these fractions. This conclusion is supported by the fact that the greatest increase in GalA content $(73-110 \%)$ was in the insoluble fractions of the PtGAUT12.1-OE biomass compared to controls, while Xyl content in these fractions was only increased 
by $14-26 \%$. Rather, the bulk of Xyl was recovered in the $1 \mathrm{M} \mathrm{KOH}$ wall fractions of the PtGAUT12.1-OE biomass compared to controls. Additional supporting evidence for structure 2 was the greater $\mathrm{Xyl} / \mathrm{GalA}$ ratio in almost all wall fractions of the PdGAUT12.1-KD compared to the control, but the similar Xyl/GalA ratios between the PtGAUT12.1-OE and control lines (Additional files 8, 9). We propose that in the PtGAUT12.1-OE lines, the PtGAUT12.1-synthesized HG was produced in higher amounts, providing more primers/anchors for the xylan to attach to. Therefore, the Xyl/GalA ratios (Additional file 8 ) would remain largely similar in the different wall extracts of the PtGAUT12.1-OE lines and the control. In the PdGAUT12.1-KD lines, however, the availability of the PdGAUT12.1-synthesized HG was markedly reduced. We propose that due to reduced amounts of HG primer/anchor, xylan synthesis would still occur but the xylan would not be integrated into the wall properly. As a result, the Xyl/GalA ratios would increase due to an overabundance of xylan compared to the PdGAUT12.1synthesized HG in almost all wall fractions. Such a result was indeed obtained in the PdGAUT12.1-KD lines compared to WT (Additional file 9). Furthermore, as noted above, some xylan would be more easily extracted and be isolated in the oxalate and carbonate fractions. Taken together, the results support the conclusion that poplar GAUT12.1 synthesizes either an HG-containing primer for xylan synthesis or an HG glycan required for proper xylan deposition, anchoring, and/or architecture in the wall, and support the hypothesis that the HG and xylan may be connected to each other by a base-sensitive covalent linkage.

\section{Conclusions}

Carbon-rich hardwood biomass has great potential as a renewable source of material for biofuels and chemicals. A major challenge for use of hardwoods as a feedstock, however, is the recalcitrance of the biomass, which hinders efficient conversion of the polysaccharides to sugars. In this research, we studied the biological function of GAUT12 in plant growth and in modifying biomass quality for saccharification by analyzing the chemical and growth phenotypes of GAUT12.1 overexpression poplar lines. The resulting PtGAUT12.1-OE transgenics had increased recalcitrance and reduced plant growth compared to controls. The stability of the introduced transgene was confirmed by maintenance of the phenotypes in a multi-year field trial. PtGAUT12.1 overexpression resulted in increased amounts of xylan and HG in the transgenic walls, a concomitant reduction in the extractability of wall material, and increased amounts of cell wall polymers being retained in the final insoluble pellets after sequential extraction of the walls with increasingly harsh solvents. The results indicate that overexpression of PtGAUT12.1 leads to wall polymers being held more tightly in the walls, and results in reduced saccharification efficiency. Overall, the phenotypes displayed by the PtGAUT12.1 overexpression lines are opposite to those previously reported for PdGAUT12.1 knockdown lines [5]. Analysis of the combined comprehensive data from the poplar GAUT12.1 overexpression and knockdown lines support the hypothesis that poplar GAUT12.1 is involved in the synthesis of a wall structure containing both HG and xylan that may be connected to each other by a base-sensitive covalent linkage.

\section{Methods}

\section{Generation of the overexpression construct and poplar transgenics lines}

A 1602 bp coding sequence of PtGAUT12.1 (Potri.001G416800) was amplified from a P. trichocarpa cDNA library via PCR using a primer pair 5'-CACCCCCGGGATGCAGCTTCATATATCGCC-3' (forward) and 5'-ACGCGTAGTTAAGATGGCCTAATATGACAGC-3' (reverse), and cloned into pENTR/D-TOPO (Life Technologies). Upon sequence verification, the fragment was transferred into a binary Gateway ${ }^{\circledR}$ destination plasmid using LR Clonase II (Life Technologies) downstream of the Arabidopsis thaliana Ubiquitin3 promoter. The resulting binary transformation vector, pAGW570 (GenBank accession number MF401557), was transformed into $A$. tumefaciens via electroporation as previously described [5]. The overexpression cassette was subsequently transformed to $P$. deltoides genotype WV94. Presence of the PtGAUT12.1-OE construct was verified using PCR from tissue culture shoots.

\section{Quantitative real time PCR and dot blot analysis}

RNA isolation and transcript analyses were performed as previously described [5]. Briefly, the shoot tip from Populus (apex), leaf (number 1-3 from top of the plants), internodes (number 1-3 from top of the plants), roots, phloem scraped from the frozen peeled bark, and xylem scraped from the debarked frozen 3-month-old stem were collected and ground to fine powder in liquid nitrogen for transcript analysis. Total RNA was isolated using a CTAB (hexadecyl-trimethylammonium bromide) method as described earlier [5] followed by removal of genomic DNA with DNase (Qiagen, Valencia, CA). Firststrand cDNA was synthesized from $1 \mu \mathrm{g}$ total RNA using the iScript cDNA Synthesis kit (Bio-Rad, Hercules, CA). Quantitative RT-PCR reactions were performed in triplicate using the $\mathrm{iQ}^{\mathrm{TM}}$ SYBR Green Supermix (Bio-Rad, Hercules, CA) and $18 S r R N A$ as the reference gene. The relative transcript expression was analyzed as described [5]. Primer sets used are as follows: GAUT12.1-F 
(5'-GGTCGAGCAAAGCCTTGGCTAGATATAGC-3') and GAUT12.1-R (5'-AGATGGCCTAATATGACAGCCCTTTA-3'), and GAUT12.2-F (5'-CATTTCAATGGTCGAGCAAAGCCTTGGC-3') and GAUT12-2-R (5'-GACAGCCCGTAATGAACTTGTCAGA-3') [5]. Note that the GAUT12.1 primer set was expected to recognize both the $P$. trichocarpa transgene and the $P$. deltoides endogenous gene due to the high level of sequence similarity between the two sequences (see "Results" section) and was previously shown to do so [5]. For dot blot analysis, cDNA was synthesized using the total RNA from vascular cambium, xylem, phloem, and leaf tissue. DNA corresponding to nucleotides 1891-2183 of PtGAUT12.1 transcript (within the $3^{\prime}$-untranslated region) was spotted onto a membrane and probed with the synthesized cDNA samples under stringent conditions as previously described [40].

\section{Plant growth conditions, growth analysis, and sample isolation}

WT and transgenic $P$. deltoides plants were grown in the greenhouse under a 16-h-light/8-h-dark cycle at 25-32 ${ }^{\circ} \mathrm{C}$, depending on the season, on Fafards 3B soil mix with osmocote, bone meal, gypsum, and dolomite/ limestone as previously described [5]. Plants were grown for 9 months. For dry weight measurement, the above ground parts (i.e., entire shoots) of 3-month-old plants were harvested, dried at $70{ }^{\circ} \mathrm{C}$ for 5 days, and weighed. Plant water status was measured as relative water content (RWC) from both WT and PtGAUT12.1-OE plants as described before [5]. For cell wall, analytical pyrolysis, and saccharification analyses, around $\sim 6 \mathrm{~cm}$ of the bottom part of the stem was harvested and the bark was peeled. The peeled stem samples were air-dried, the pith removed, the remaining tissues milled to a particle size of 20 mesh $(0.85 \mathrm{~mm})$, and the ground samples used for analyses [5].

For the field study, transgenic and control plants were grown in Claxton (GA-30417, USA, $32^{\circ} 9^{\prime} 39^{\prime \prime} \mathrm{N}$ $81^{\circ} 54^{\prime} 31^{\prime \prime} \mathrm{W}$, with a humid subtropical climate) (Additional file 11). Stem radial diameter was measured on 2.8-year-old field-grown transgenic and controls plants. For cell wall analysis, $\sim 15 \mathrm{~cm}$ of the bottom part of the debarked stem was harvested and air-dried, the pith removed, milled to a particle size of 20 mesh $(0.85 \mathrm{~mm})$, and the ground samples used for analysis.

\section{Cell wall analysis}

AIR and fractionated cell walls from ground biomass were prepared as previously described [5]. Glycosyl residue composition analysis of the AIR $(\sim 2 \mathrm{mg})$ and cell wall fractions $(100-300 \mu \mathrm{g})$ from WT and PtGAUT12.1-OE samples was performed by combined gas chromatography/mass spectrometry (GC/MS) of the per-O-trimethylsilyl (TMS) derivatives of the monosaccharide methyl glycosides produced from the sample by acidic methanolysis as described previously [5, 41, 42]. For glycosyl linkage analysis, samples were permethylated, reduced, re-permethylated, depolymerized, reduced, and acetylated; and the resulting partially methylated alditol acetates (PMAAs) were analyzed by gas chromatography-mass spectrometry (GC-MS) as described [5, 43].

\section{Lignin analysis and saccharification assay}

The National Renewable Energy Laboratory (NREL) high-throughput pyrolysis molecular beam mass spectrometry (MBMS) method was used to quantify lignin content and S/G lignin monomer ratio from WT and PtGAUT12.1-OE biomass as previously described [5, $44,45]$. For the saccharification assay, the NREL highthroughput thermochemical pretreatment and enzymatic hydrolysis sugar release assay was carried out as previously described $[5,46,47]$.

\section{Glycome profiling}

Sequential cell walls extracted fractions from Populus WT and PtGAUT12.1-OE lines were subjected to glycome profiling analysis as described $[5,25]$. The presence of epitopes recognized by Enzyme-Linked Immunosorbent Assay (ELISA)-based monoclonal antibodies (mAb) screenings were represented as heat maps.

\section{Microscopy}

Tissue fixation and embedding, maceration of xylem, and microscopy analyses were performed as described [5].

\section{Statistical analysis}

Statistical analysis was performed using Statistica 5.0. The significance of differences between control and transgenic samples was analyzed using a one-way ANOVA followed by Tukey's multiple comparison test.

\section{Additional files}

Additional file 1. a Glucose, $\mathbf{b}$ xylose, and $\mathbf{c}$ total sugar release from $P$. deltoides wild-type (WT), vector control and PtGAUT12-OE lines. $n=25$ for WT, $n=10-15$ for each vector control and PtGAUT12-OE lines. Signifcance $P$ values are expressed as ${ }^{*} P<0.05,{ }^{* *} P<0.001$ by one-way analysis of variance (ANOVA) followed by Tukey's multiple comparison test using Statistica 5.0.

Additional file 2. Total lignin content and $S / G$ ratio of $P$. deltoides wildtype (WT), vector control and PtGAUT12.1-OE lines. Values are mean \pm SE, $n=25$ for WT, $n=10-15$ for vector control (V. Control-1-8) and

PtGAUT12.1-OE lines (AB29.1-AB29.13). In bold and denoted with a star are transgenic values that are significantly different from WT and vector 
control lines at $P<0.05$, as determined by one-way analysis of variance (ANOVA) followed by Tukey's multiple comparison test using Statistica 5.0.

Additional file 3. Plant height and diameter of three-month-old P. deltoides wild-type (WT), vector control and PtGAUT12.1-OE lines. Values are mean $\pm \mathrm{SE}, n=25$ for WT, $n=10-15$ for vector control (V. Control-1-8) and PtGAUT12.1-OE lines (AB29.1-AB29.13). Transgenic values that are significantly different from WT and vector control lines are in bold and denoted with one star $(P<0.05)$ or two stars $(P<0.001)$, as determined by one-way analysis of variance (ANOVA) followed by Tukey's multiple comparison test using Statistica 5.0

Additional file 4. Correlation between the total GAUT12.1 transcript expression and plant growth in P. deltoides PtGAUT12.1-OE transgenic and control lines. $\mathbf{a}$ Height and $\mathbf{b}$ radial diameter of 3-month-old greenhousegrown poplar plants are plotted against the transcript expression level. Blue diamonds - WT; black diamonds - vector controls; red diamondsPtGAUT12.1-OE lines (please note that on the graphs the WT blue diamonds may be obscured by the vector control black diamonds). $n=25$ for WT, $n=10-15$ for vector control and PtGAUT12.1-OE lines.

Additional file 5. Leaf phenotypes of 3-month-old, greenhouse-grown P. deltoides PtGAUT12.1-OE lines compared to controls. a Comparison of leaves (the sixth leaf from apex) from P. deltoides WT, vector control, and PtGAUT12.1-OE plants. $\mathbf{b}$ Length and $\mathbf{c}$ width of leaves of different developmental stages from 3-month-old plants. Every third leaf from the apex of ten plants was measured. $\mathbf{d}$ Developing (10th leaf from apex) and $\mathbf{e}$ fully expanded (20th leaf from apex) leaf areas measured from five plants. f The relative water content (RWC) of WT and PtGAUT12.1-OE lines. Error bars represent $S E .{ }^{*} P<0.05,{ }^{* *} P<0.001 . \mathbf{g}$, h Correlation between RWC at $72 \mathrm{~h}$ and fully expanded leaf area of WT and P. deltoides PdGAUT12.1-KD (g) and PtGAUT12.1-OE (h) lines.

Additional file 6. Glycosyl residue composition of (a) alcohol insoluble residue (AIR) and $(\mathbf{b}-\mathbf{h})$ wall fractions from stems of field-grown P. deltoides control and PtGAUT12.1-OE transgenic plants. Wall fractions were prepared by sequential extraction of AIR using increasingly harsh reagents: (b) $50 \mathrm{mM}$ ammonium oxalate, (c) $50 \mathrm{mM} \mathrm{Na}_{2} \mathrm{CO}_{3}$ (d) $1 \mathrm{M} \mathrm{KOH}$, (e) $4 \mathrm{M} \mathrm{KOH}$, (f) $100 \mathrm{mM}$ sodium chlorite (chlorite) and (g) $4 \mathrm{M} \mathrm{KOH}$ post-chlorite (4 M $\mathrm{KOH} \mathrm{PC}$ ). (h) The insoluble pellet remaining after all the extractions. Glycosyl residue composition was determined by GC-MS of trimetylsilyl (TMS) derivatives. Data are mean \pm SE of three biological and two technical replicates, $n=5$. ${ }^{*} P<0.05,{ }^{*} P<<0.001$.

Additional file 7. Mass of AIR, total material recovered in sequential AIR extracts and in the insoluble pellet from P. deltoides PtGAUT12.1-OE lines compared to controls. a Mass of AIR extracted per gram of ground dry stem tissues of PtGAUT12.1-OE and control lines. b-g Amount of material recovered in wall fractions extracted by $\mathbf{b} 50 \mathrm{mM}$ ammonium oxalate, c $50 \mathrm{mM}$ sodium carbonate, d $1 \mathrm{M} \mathrm{KOH}$, e $4 \mathrm{M} \mathrm{KOH}, \mathbf{f} 100 \mathrm{mM}$ sodium chlorite, $\mathbf{g} 4 \mathrm{M} \mathrm{KOH}$ post chlorite $(\mathrm{PC})$. $\mathbf{h}$ Total amount of material recovered in all wall fractions combined. $\mathbf{i}$ Amount of material remaining in the insoluble pellet, after all the extractions. Data in panels $\mathbf{b}$ through $\mathbf{i}$ are average $\mathrm{mg}$ extract per gram AIR $\pm \mathrm{SE}, n=4$. Significance $P$ values are expressed as ${ }^{*} P<0.05,{ }^{*} P<0.001$.

Additional file 8. Glycosyl residue composition and total carbohydrate of cell wall extracts from stem tissues of 9-month-old, greenhouse-grown P. deltoides WT and PtGAUT12.1-OE lines (AB29.2, AB29.7, and AB29.12).

Data are average $\mu \mathrm{g}$ amount of each designated monosaccharide per $\mathrm{mg}$ alcohol insoluble residue (AIR), measured by GC-MS of tetramethylsilane (TMS) derivatives. Wall extracts are from sequential extractions of AIR using $50 \mathrm{mM}$ ammonium oxalate, $50 \mathrm{mM}$ sodium carbonate, $1 \mathrm{M} \mathrm{KOH}$, $4 \mathrm{M} \mathrm{KOH}, 100 \mathrm{mM}$ sodium chlorite (chlorite), $4 \mathrm{M} \mathrm{KOH}$ post-chlorite (PC), and the final residual insoluble pellet. Values in bold are those of the transgenic lines that are significantly different from WT values at ${ }^{*} P<0.05$ and ${ }^{* *} P<0.001$ significant levels (one-way ANOVA followed by Tukey's multiple comparison test).

Additional file 9. Glycosyl residue composition and total carbohydrate of cell wall extracts from stem tissues of 9-month-old, greenhouse-grown
P. deltoides WT and PdGAUT12.1-KD lines (AB30.1, AB30.3, AB30.8 and AB30.11). Data are average $\mu \mathrm{g}$ amount of each designated monosaccharide per mg alcohol insoluble residue (AIR), measured by GC-MS of tetramethylsilane (TMS) derivatives. Wall extracts are from sequential extractions of AIR using $50 \mathrm{mM}$ ammonium oxalate, $50 \mathrm{mM}$ sodium carbonate, $1 \mathrm{M} \mathrm{KOH}, 4 \mathrm{M} \mathrm{KOH}, 100 \mathrm{mM}$ sodium chlorite (chlorite), $4 \mathrm{M} \mathrm{KOH}$ post-chlorite $(\mathrm{PC})$, and the final residual insoluble pellet. The data, except for those of the insoluble wall fractions, were previously reported in mol\% and mass ( $\mu \mathrm{g}$ sugar/mg extract) [5]. Values in bold are those of the transgenic lines that are significantly different from WT values at $* P<0.05$ and ${ }^{* *} P<0.001$ significant levels (one-way ANOVA followed by Tukey's multiple comparison test).

Additional file 10. Glycosyl linkage analysis of the residual insoluble cell wall that remained after sequential extractions of AIR from P. deltoides WT, PdGAUT12.1-KD, and PtGAUT12.1-OE lines. Data are in mol percentages, provided by CCRC Analytical Services.

Additional file 11. Field design of the multi-year field trial of $P$. deltoides PtGAUT12.1-OE and control lines. The field was divided into three replicate plots, each contained completely randomized genotype subplots. Each subplot contained two vegetatively propagated clonal trees, planted $3 \mathrm{~m} \times 3 \mathrm{~m}$ apart from each other and from other trees in the neighboring subplots. Border trees were also planted surrounding the whole plot to control for shading effects. Grey boxes represent subplots containing transgenics lines with genotypes other than the PtGAUT12.1-OE, vector control, and WT lines.

\section{Abbreviations}

AIR: alcohol insoluble residue; GalA: galacturonic acid; GAUT1: GAlactUronosylTransferase1; GAUT12: GAlactUronosylTransferase12; GlcA: glucuronic acid; GT8: glycosyltransferase family 8; GX: glucuronoxylan; HG: homogalacturonan; MBMS: molecular beam mass spectrometry; MeGlcA: methyl glucuronic acid; RG: rhamnogalacturonan; TMS: trimethylsilyl; WT: wild type.

\section{Authors' contributions}

AKB planned and designed the research, participated in all aspects of the study, oversaw plant phenotyping, plant growth, line selection, tissue handling and distribution, expression study, carried out plant physiological and cell wall analyses, and wrote the manuscript. MAA analyzed data and wrote the manuscript. SP and MGH performed and analyzed the glycome profiling analysis. RA contributed to cell wall extraction. XY identified Populus genes based on provided Arabidopsis homolog and designed the DNA construct. KW carried out molecular cloning and production of the overexpression plasmid. CC performed Populus transformation and propagated transgenic plants. SSM and DR participated in growing, sampling, and analysis of the plants. LT contributed to cell wall analysis and wrote the manuscript. IGA and KH contributed to the identification of Populus genes and the growing of plants. RWS conducted high-throughput pyrolysis molecular beam mass spectrometry (py-MBMS) lignin assays. GBT performed high-throughput recalcitrance pipeline through BESC and analyzed sugar release values. AZ coordinated analysis of samples through the BioEnergy Science Center (BESC) high-throughput molecular beam mass spectrometry (MBMS) and saccharification pipelines. MFD developed and provided leadership for MBMS pipeline through BESC. SRD guided overall high-throughput saccharification pipeline through BESC and provided data analysis. DM conceived the study, coordinated research, contributed to interpretation of results, and helped draft and finalize the manuscript. All authors read and approved the final manuscript.

\section{Author details}

${ }^{1}$ Department of Biochemistry and Molecular Biology, University of Georgia, Athens, GA 30602, USA. ${ }^{2}$ Department of Plant Biology, University of Georgia, Athens, GA 30602, USA. ${ }^{3}$ Complex Carbohydrate Research Center, University of Georgia, 315 Riverbend Rd., Athens, GA 30602-4712, USA. ${ }^{4}$ DOE-BioEnergy Science Center (BESC), Oak Ridge National Laboratory, Oak Ridge, TN 37831, 
USA. ${ }^{5}$ Bioscience Division, Oak Ridge National Laboratory, Oak Ridge, TN 37831, USA. ${ }^{6}$ ArborGen, Inc., 2011 Broadbank Ct., Ridgeville, SC 29472, USA. ${ }^{7}$ National Renewable Energy Laboratory, Golden, CO 80401-3305, USA. ${ }^{8}$ Present Address: Mascoma LLC (Lallemand Inc.), 67 Etna Rd., Lebanon, NH 03766, USA. ${ }^{9}$ Present Address: South Georgia State College, 100 West College Park Dr., Douglas, GA 31533, USA. ${ }^{10}$ Present Address: Nuclear Materials Science, Los Alamos National Laboratory, P.O. Box 1663, Los Alamos, NM 87545-1663, USA.

11 Present Address: Nu Mark LLC, 6601 W. Broad St., Richmond, VA 23230, USA.

\section{Acknowledgements}

We thank Will Rottmann for leading the Populus transformation, Rick Nelson for directing the BESC transformation pipeline, Lee Gunter for validation of Populus constructs, and CCRC Analytical Services for glycosyl residue linkage analysis. We also thank Sheilah Dixon Huckabee for administrative assistance, and Reggie Millwood for assistance with the USDA APHIS BRS permit regulations. The work was primarily supported by BioEnergy Science Center Grant DE-PS02-06ER64304, and partially by the Center for Bioenergy Innovation. The BioEnergy Science Center and the Center for Bioenergy Innovation are US Department of Energy Bioenergy Research Centers supported by the Office of Biological and Environmental Research in the Department of Energy's Office of Science. The research was also partially funded by the Department of Energy Center Grant DE-SC0015662. The CCRC series of plant cell wall glycan-directed antibodies were generated with the support of the US National Science Foundation Plant Genome Program (Grants DBI-0421683 and IOS-0923992).

\section{Competing interests}

The strategy to produce improved biomass as described in this paper has been included in a patent application. Cassandra Collins and Kim Winkeler are employees of ArborGen Inc., a global provider of conventional and next generation plantation tree seedling products for the forestry industry.

\section{Availability of data and materials}

All data generated or analyzed during this study are included in this published article [and its Additional files].

\section{Consent for publication}

Not applicable.

\section{Ethics approval and consent to participate}

Not applicable.

\section{Funding}

The generation of the CCRC series of plant cell wall glycan-directed monoclonal antibodies used in this work was supported by the US National Science Foundation Plant Genome Program (DBI-0421683 and IOS-0923992). The research was funded by The BioEnergy Science Center (BESC) Grant DE-PS02-06ER64304, and partially by the Department of Energy Center Grant DE-SC0015662 and by the Center for Bioenergy Innovation. The BioEnergy Science Center and the Center for Bioenergy Innovation are US Department of Energy Bioenergy Research Centers supported by the Office of Biological and Environmental Research in the DOE Office of Science.

\section{Publisher's Note}

Springer Nature remains neutral with regard to jurisdictional claims in published maps and institutional affiliations.

Received: 18 October 2017 Accepted: 18 December 2017 Published online: 17 January 2018

\section{References}

1. Himmel ME, Ding SY, Johnson DK, Adney WS, Nimlos MR, Brady JW, et al. Biomass recalcitrance: engineering plants and enzymes for biofuels production. Science. 2007;315:804-7.

2. Lynd LR, Laser MS, Bransby D, Dale BE, Davison B, Hamilton R, et al. How biotech can transform biofuels. Nat Biotechnol. 2008;26:169-72.

3. Bonan GB. Forests and climate change: forcings, feedbacks, and the climate benefits of forests. Science. 2008;320:1444-9.
4. Séquin A. How could forest trees play an important role as feedstock for bioenergy production? Curr Opin Environ Sustain. 2011;3:90-4.

5. Biswal AK, Hao Z, Pattathil S, Yang X, Winkeler K, Collins C, et al. Downregulation of GAUT12 in Populus deltoides by RNA silencing results in reduced recalcitrance, increased growth and reduced xylan and pectin in a woody biofuel feedstock. Biotechnol Biofuels. 2015;8:41.

6. Mellerowicz EJ, Sundberg B. Wood cell walls: biosynthesis, developmental dynamics and their implications for wood properties. Curr Opin Plant Biol. 2008;11:293-300.

7. Mizrachi E, Mansfield SD, Myburg AA. Cellulose factories: advancing bioenergy production from forest trees. New Phytol. 2012;194:54-62.

8. Ragauskas AJ, Beckham GT, Biddy MJ, Chandra R, Chen F, Davis MF, et al. Lignin valorization: improving lignin processing in the biorefinery. Science. 2014;344:1246843.

9. Cantarel BL, Coutinho PM, Rancurel C, Bernard T, Lombard V, Henrissat B. The carbohydrate-active enzymes database (CAZy): an expert resource for glycogenomics. Nucleic Acids Res. 2009;37:D233-8.

10. Sterling JD, Atmodjo MA, Inwood SE, Kumar Kolli VS, Quigley HF, Hahn MG, et al. Functional identification of an Arabidopsis pectin biosynthetic homogalacturonan galacturonosyltransferase. Proc Natl Acad Sci USA. 2006;103:5236-41.

11. Brown DM, Zeef LA, Ellis J, Goodacre R, Turner SR. Identification of novel genes in Arabidopsis involved in secondary cell wall formation using expression profiling and reverse genetics. Plant Cell. 2005;17:2281-95.

12. Persson S, Wei H, Milne J, Page GP, Somerville CR. Identification of genes required for cellulose synthesis by regression analysis of public microarray data sets. Proc Natl Acad Sci USA. 2005;102:8633-8.

13. Pena MJ, Zhong R, Zhou GK, Richardson EA, O'Neill MA, Darvill AG, et al. Arabidopsis irregular xylem 8 and irregular xylem9: implications for the complexity of glucuronoxylan biosynthesis. Plant Cell. 2007;19:549-63.

14. Persson S, Caffall KH, Freshour G, Hilley MT, Bauer S, Poindexter P, et al. The Arabidopsis irregular xylem 8 mutant is deficient in glucuronoxylan and homogalacturonan, which are essential for secondary cell wall integrity. Plant Cell. 2007;19:237-55.

15. Hao Z, Avci U, Tan L, Zhu X, Glushka J, Pattathil S, et al. Loss of arabidopsis GAUT12/IRX8 causes anther indehiscence and leads to reduced $G$ lignin associated with altered matrix polysaccharide deposition. Front Plant Sci. 2014;5:357.

16. Brown DM, Goubet F, Wong VW, Goodacre R, Stephens E, Dupree P, et al. Comparison of five xylan synthesis mutants reveals new insight into the mechanisms of xylan synthesis. Plant J. 2007;52:1154-68.

17. Petersen PD, Lau J, Ebert B, Yang F, Verhertbruggen Y, Kim JS, et al. Engineering of plants with improved properties as biofuels feedstocks by vessel-specific complementation of xylan biosynthesis mutants. Biotechnol Biofuels. 2012;5:84.

18. Biswal AK, Atmodjo MA, Li M, Baxter HL, Yoo CG, Pu Y, et al. Sugar release and growth of biofuel crops are improved by downregulation of pectin biosynthesis. Nat Biotechnol (in press).

19. Zhou GK, Zhong R, Himmelsbach DS, McPhail BT, Ye ZH. Molecular characterization of PoGT8D and PoGT43B, two secondary wall-associated glycosyltransferases in poplar. Plant Cell Physiol. 2007;48:689-99.

20. Lee C, O'Neill MA, Tsumuraya Y, Darvill AG, Ye ZH. The irregular xylem 9 mutant is deficient in xylan xylosyltransferase activity. Plant Cell Physiol. 2007;48:1624-34.

21. Atmodjo MA, Sakuragi Y, Zhu X, Burrell AJ, Mohanty SS, Atwood JA 3rd, et al. Galacturonosyltransferase (GAUT) 1 and GAUT7 are the core of a plant cell wall pectin biosynthetic homogalacturonan:galacturonosyltran sferase complex. Proc Natl Acad Sci USA. 2011;108:20225-30.

22. Li Q, Min D, Wang JP, Peszlen I, Horvath L, Horvath B, et al. Downregulation of glycosyltransferase $8 \mathrm{D}$ genes in Populus trichocarpa caused reduced mechanical strength and xylan content in wood. Tree Physiol. 2011;31:226-36.

23. Lee C, Teng Q, Zhong R, Ye ZH. Molecular dissection of xylan biosynthesis during wood formation in poplar. Mol Plant. 2011;4:730-47.

24. Mizrachi E, Verbeke L, Christie N, Fierro AC, Mansfield SD, Davis MF, et al. Network-based integration of systems genetics data reveals pathways associated with lignocellulosic biomass accumulation and processing. Proc Natl Acad Sci USA. 2017;114:1195-200.

25. Pattathil S, Avci U, Miller JS, Hahn MG. Immunological approaches to plant cell wall and biomass characterization: glycome profiling. Methods Mol Biol. 2012;908:61-72. 
26. Pattathil S, Avci U, Zhang T, Cardenas CL, Hahn MG. Immunological approaches to biomass characterization and utilization. Front Bioeng Biotechnol. 2015;3:173.

27. Biswal AK, Tan L, Atmodjo MA, DeMartini J, Gelineo-Albersheim I, Hunt $\mathrm{K}$, et al. Comparison of four glycosyl residue composition methods for effectiveness in detecting sugars from cell walls of dicot and grass tissues. Biotechnol Biofuels. 2017:10:182

28. Tan L, Eberhard S, Pattathil S, Warder C, Glushka J, Yuan C, et al. An Arabidopsis cell wall proteoglycan consists of pectin and arabinoxylan covalently linked to an arabinogalactan protein. Plant Cell. 2013;25:270-87.

29. Pattathil S, Avci U, Baldwin D, Swennes AG, McGill JA, Popper Z, et al. A comprehensive toolkit of plant cell wall glycan-directed monoclonal antibodies. Plant Physiol. 2010;153:514-25.

30. Ruprecht C, Bartetzko MP, Senf D, Dallabernardina P, Boos I, Andersen MCF, et al. A synthetic glycan microarray enables epitope mapping of plant cell wall glycan-directed antibodies. Plant Physiol. 2017;175(3):1094-104.

31. Schmidt D, Schuhmacher F, Geissner A, Seeberger PH, Pfrengle F. Automated synthesis of arabinoxylan-oligosaccharides enables characterization of antibodies that recognize plant cell wall glycans. Chemistry. 2015;21:5709-13.

32. Clausen MH, Willats WG, Knox JP. Synthetic methyl hexagalacturonate hapten inhibitors of anti-homogalacturonan monoclonal antibodies LM7, JIM5 and JIM7. Carbohydr Res. 2003;338:1797-800.

33. Mohr H, Schopfer P. Plant physiology. Berlin: Springer; 1995.

34. Caffall KH, Pattathil S, Phillips SE, Hahn MG, Mohnen D. Arabidopsis thaliana T-DNA mutants implicate GAUT genes in the biosynthesis of pectin and xylan in cell walls and seed testa. Mol Plant. 2009;2:1000-14.

35. Wang T, Hong M. Solid-state NMR investigations of cellulose structure and interactions with matrix polysaccharides in plant primary cell walls. J Exp Bot. 2016;67:503-14.

36. Wang T, Park YB, Cosgrove DJ, Hong M. Cellulose-pectin spatial contacts are inherent to never-dried Arabidopsis primary cell walls: evidence from Solid-State Nuclear Magnetic Resonance. Plant Physiol. 2015;168:871-84.

37. Wang T, Zabotina $\mathrm{O}$, Hong M. Pectin-cellulose interactions in the Arabidopsis primary cell wall from two-dimensional magic-angle-spinning solid-state nuclear magnetic resonance. Biochemistry. 2012;51:9846-56.

38. Aspeborg H, Schrader J, Coutinho PM, Stam M, Kallas A, Djerbi S, et al. Carbohydrate-active enzymes involved in the secondary cell wall biogenesis in hybrid aspen. Plant Physiol. 2005;137:983-97.
39. Mellerowicz EJ, Baucher M, Sundberg B, Boerjan W. Unravelling cell wall formation in the woody dicot stem. Plant Mol Biol. 2001;47:239-74.

40. Siedlecka A, Wiklund S, Peronne MA, Micheli F, Lesniewska J, Sethson I, et al. Pectin methyl esterase inhibits intrusive and symplastic cell growth in developing wood cells of Populus. Plant Physiol. 2008;146:554-65.

41. York WS, Darvill AG, MCNeil M, Stevenson TT, Albersheim P. Isolation and characterization of plant cell walls and cell wall components. Methods Enzymol. 1985;118:3-40.

42. Santander J, Martin T, Loh A, Pohlenz C, Gatlin DM 3rd, Curtiss R 3rd. Mechanisms of intrinsic resistance to antimicrobial peptides of Edwardsiella ictaluri and its influence on fish gut inflammation and virulence. Microbiology. 2013;159:1471-86.

43. Heiss C, Klutts JS, Wang Z, Doering TL, Azadi P. The structure of Cryptococcus neoformans galactoxylomannan contains beta-D-glucuronic acid. Carbohydr Res. 2009;344:915-20.

44. Evans RJ, Milne TA. Molecular characterization of the pyrolysis of biomass. Energy Fuels. 1987;1:123-37.

45. Sykes R, Yung M, Novaes E, Kirst M, Peter G, Davis M. High-throughput screening of plant cell-wall composition using pyrolysis molecular beam mass spectroscopy. In: Mielenz JR, editor. Biofuels: methods and protocols, methods in molecular biology, vol. 581. New York: Humana Press; 2009. p. 169-83.

46. Decker SR, Brunecky R, Tucker MP, Himmel ME, Selig MJ. High-throughput screening techniques for biomass conversion. Bioenerg Res. 2009;2:179-92.

47. Decker SR, Carlile M, Selig MJ, Doeppke C, Davis M, Sykes R, et al. Reducing the effect of variable starch levels in biomass recalcitrance screening. In: Himmel ME, editor. Biomass conversion methods in molecular biology, vol. 908. New York: Humana Press; 2012. p. 181-95.

48. Tamura K, Peterson D, Peterson N, Stecher G, Nei M, Kumar S. MEGA5: molecular evolutionary genetics analysis using maximum likelihood, evolutionary distance, and maximum parsimony methods. Mol Biol Evol. 2011;28:2731-9.

49. Krogh A, Larsson B, von Heijne G, Sonnhammer EL. Predicting transmembrane protein topology with a hidden Markov model: application to complete genomes. J Mol Biol. 2001;305:567-80.

50. Hertzberg M, Aspeborg H, Schrader J, Andersson A, Erlandsson R, Blomqvist K, et al. A transcriptional roadmap to wood formation. Proc Natl Acad Sci USA. 2001;98:14732-7.

\section{Submit your next manuscript to BioMed Central and we will help you at every step:}

- We accept pre-submission inquiries

- Our selector tool helps you to find the most relevant journal

- We provide round the clock customer support

- Convenient online submission

- Thorough peer review

- Inclusion in PubMed and all major indexing services

- Maximum visibility for your research

Submit your manuscript at www.biomedcentral.com/submit
BioMed Central 